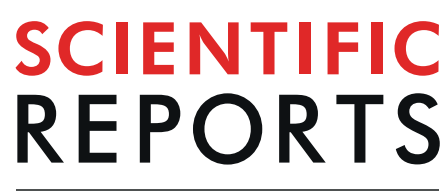

natureresearch

Check for updates

\title{
Relationship between headaches and tinnitus in a Swedish study
}

\author{
Alessandra Lugo ${ }^{1,9}$, Niklas K. Edvall 2,9 , Andra Lazar ${ }^{3}$, Golbarg Mehraei ${ }^{4}$, \\ Jose-Antonio Lopez-Escamez ${ }^{5,6}$, Jan Bulla ${ }^{7,8}$, Inger Uhlen ${ }^{3}$, Barbara Canlon ${ }^{2}$, \\ Silvano Gallus ${ }^{1} \&$ Christopher R. Cederroth ${ }^{2}$
}

The heterogeneity of tinnitus is likely accounting for the lack of effective treatment approaches. Headaches have been related to tinnitus, yet little is known on how headaches impact tinnitus. We use cross-sectional data from the Swedish Tinnitus Outreach Project to i) evaluate the association between headaches and tinnitus ( $n=1,984$ cases and 1,661 controls) and ii) investigate the phenotypic characteristics of tinnitus subjects with tinnitus $(n=660)$ or without $(n=1,879)$ headaches. In a multivariable logistic regression model, headache was significantly associated with any tinnitus (odds ratio, $\mathrm{OR}=2.61$ ) and more so with tinnitus as a big problem (as measured by the tinnitus functional index, $\mathrm{TFI} \geq 48 ; \mathrm{OR}=5.63$ ) or severe tinnitus (using the tinnitus handicap inventory, $\mathrm{THI} \geq 58 ; \mathrm{OR}=4.99$ ). When focusing on subjects with tinnitus, the prevalence of headaches was $26 \%$ and reached $40 \%$ in subjects with severe tinnitus. A large number of socioeconomic, phenotypic and psychological characteristics differed between headache and non-headache subjects with any tinnitus. With increasing tinnitus severity, fewer differences were found, the major ones being vertigo, neck pain and other pain syndromes, as well as stress and anxiety. Our study suggests that headaches could contribute to tinnitus distress and potentially its severity.

Subjective tinnitus is the perception of sounds in absence of external stimuli. Its prevalence ranges from 5-43\%, and for about one in ten with tinnitus, the impact is such that individuals seek medical help ${ }^{1}$. This so-called clinically significant tinnitus is strongly associated with anxiety, depression and stress ${ }^{2-6}$. Recent studies revealed an increased risk in suicide attempts in subjects with tinnitus ${ }^{7,8}$. The combination of these psychological comorbidities with tinnitus has a non-negligible impact on quality of life ${ }^{3}$. Available treatments are of limited efficacy ${ }^{9-11}$, subsequently leading to substantial healthcare costs ${ }^{12}$. It is now agreed that the heterogeneity of tinnitus could be grounds to treatment failures ${ }^{13}$, which has led to research programs aimed at identifying means of classifying tinnitus subtypes ${ }^{14}$. For instance, hyperacusis ${ }^{15}$ and temporomandibular complaints ${ }^{16,17}$ have been proposed as potential criteria for subtyping tinnitus.

Headaches have also been recently suggested as an important co-factor for tinnitus subtyping ${ }^{18}$, but there is limited knowledge on how headaches impact tinnitus. Similar to tinnitus, headaches are very heterogeneous. Migraine is a common primary headache disorder that can be experienced with or without aura, which are defined by episodes of transient focal neurological symptoms. The neural mechanisms of migraine have been reviewed elsewhere ${ }^{19}$. Recent studies reveal a high prevalence of headache (26-56\%) in children, adolescents and patients with tinnitus ${ }^{18,20,21}$. Indeed, cross-sectional studies show that headache is associated with tinnitus ${ }^{22,23}$, and this association is greater in presence of migraine headache with aura ${ }^{24}$. As headache are very prevalent in the population, the co-occurrence of tinnitus and headache or migraine could be coincidental. However, two longitudinal studies show that a history of migraine is a risk factor for the development of tinnitus ${ }^{25,26}$.

The pathophysiology of tinnitus is thought to derive from a failure to adapt to missing sensory information ${ }^{27}$. Concomitant with hearing loss, the brain attempts to compensate for the diminished sensory input causing an

${ }^{1}$ Department of Environmental Health Sciences, Istituto di Ricerche Farmacologiche Mario Negri IRCCS, Milan, Italy. ${ }^{2}$ Laboratory of Experimental Audiology, Department of Physiology and Pharmacology, Karolinska Institutet, 171 77, Stockholm, Sweden. ${ }^{3}$ Hörsel och balansmottagningen, Karolinska Universitetssjukhuset, Stockholm, Sweden. ${ }^{4}$ Decibel Therapeutics Inc., Boston, USA. ${ }^{5}$ Otology \& Neurotology Group, Department of Genomic Medicine, Pfizer - Universidad de Granada - Junta de Andalucía Centro de Genómica e Investigación Oncológica (GENYO), PTS, Avenida de la llustración 114, 18016, Granada, Spain. ${ }^{6}$ Department of Otolaryngology, Hospital Universitario Virgen de las Nieves, Instituto de Investigacion Biosanitaria ibs.GRANADA, Granada, Spain. ${ }^{7}$ University of Bergen, Bergen, Norway. ${ }^{8}$ University of Regensburg, Regensburg, Germany. ${ }^{9}$ These authors contributed equally: Alessandra Lugo and Niklas K. Edvall. ${ }_{e}$-mail: christopher.cederroth@ki.se 


\begin{tabular}{|c|c|c|c|c|c|c|c|c|c|c|}
\hline & \multirow{2}{*}{\begin{tabular}{|l} 
No tinnitus \\
N (\%)
\end{tabular}} & \multicolumn{3}{|l|}{ Any tinnitus } & \multicolumn{3}{|c|}{$\begin{array}{l}\text { Tinnitus as a big problem } \\
(\mathrm{TFI} \geq 48)\end{array}$} & \multicolumn{3}{|c|}{ Severe tinnitus (THI $\geq 58$ ) } \\
\hline & & $\mathrm{N}(\%)$ & \begin{tabular}{|l|} 
OR* \\
$(95 \%$ \\
CI $)$
\end{tabular} & \begin{tabular}{|l|}
$\mathrm{OR}^{\circ}$ \\
$(\mathbf{9 5} \%$ \\
$\mathrm{CI})$
\end{tabular} & N (\%) & $\begin{array}{l}\text { OR* } \\
(95 \% \\
\text { CI) }\end{array}$ & \begin{tabular}{|l|}
$\mathbf{O R}^{\circ}$ \\
$(\mathbf{9 5} \%$ \\
$\mathrm{CI})$
\end{tabular} & N (\%) & $\begin{array}{l}\text { OR* } \\
(95 \% \\
\text { CI })\end{array}$ & $\begin{array}{l}\mathrm{OR}^{\circ} \\
(\mathbf{9 5} \% \\
\mathrm{CI})\end{array}$ \\
\hline \multicolumn{11}{|c|}{ Both sexes } \\
\hline Total & $1661(100.0)$ & $1984(100.0)$ & - & - & $263(100.0)$ & - & - & $157(100.0)$ & - & - \\
\hline \multicolumn{11}{|c|}{ Headache } \\
\hline No & $1421(85.6)$ & $1458(73.5)$ & $1^{\wedge}$ & $1^{\wedge}$ & $156(59.3)$ & $1^{\wedge}$ & $1^{\wedge}$ & 94 (59.9) & $1^{\wedge}$ & $1^{\wedge}$ \\
\hline Yes & $240(14.4)$ & $526(26.5)$ & $\begin{array}{l}2.61 \\
(2.19- \\
3.12)\end{array}$ & $\begin{array}{l}2.19 \\
(1.81- \\
2.64)\end{array}$ & $107(40.7)$ & $\begin{array}{l}5.63 \\
(4.10- \\
7.72)\end{array}$ & $\begin{array}{l}4.29 \\
(2.96- \\
6.22)\end{array}$ & $63(40.1)$ & $\begin{array}{l}4.99 \\
(3.41- \\
7.32)\end{array}$ & $\begin{array}{l}3.80 \\
(2.43- \\
5.94)\end{array}$ \\
\hline \multicolumn{11}{|l|}{ Men } \\
\hline Total & $563(100.0)$ & $947(100.0)$ & - & - & $127(100.0)$ & - & - & $71(100.0)$ & - & - \\
\hline \multicolumn{11}{|c|}{ Headache } \\
\hline No & $524(93.1)$ & 768 (81.1) & $1^{\wedge}$ & $1^{\wedge}$ & 87 (68.5) & $1^{\wedge}$ & $1^{\wedge}$ & 46 (64.8) & $1^{\wedge}$ & $1^{\wedge}$ \\
\hline Yes & $39(6.9)$ & $179(18.9)$ & $\begin{array}{l}3.21 \\
(2.22- \\
4.64) \\
\end{array}$ & \begin{tabular}{|l|}
2.64 \\
$(1.80-$ \\
$3.87)$ \\
\end{tabular} & $40(31.5)$ & $\begin{array}{l}7.28 \\
(4.19- \\
12.63) \\
\end{array}$ & $\begin{array}{l}6.09 \\
(3.26- \\
11.35) \\
\end{array}$ & $25(35.2)$ & $\begin{array}{l}8.14 \\
(4.22- \\
15.7) \\
\end{array}$ & $\begin{array}{l}7.47 \\
(3.55- \\
15.7) \\
\end{array}$ \\
\hline \multicolumn{11}{|c|}{ Women } \\
\hline Total & $1098(100.0)$ & $1034(100.0)$ & - & - & $135(100.0)$ & - & -- & $85(100.0)$ & - & - \\
\hline \multicolumn{11}{|c|}{ Headache } \\
\hline No & $897(81.7)$ & $689(66.6)$ & $1^{\wedge}$ & $1^{\wedge}$ & $68(50.4)$ & $1^{\wedge}$ & $1^{\wedge}$ & $47(55.3)$ & $1^{\wedge}$ & $1^{\wedge}$ \\
\hline Yes & $201(18.3)$ & $345(33.4)$ & $\begin{array}{l}2.49 \\
(2.03- \\
3.06)\end{array}$ & $\begin{array}{l}2.09 \\
(1.68- \\
2.61)\end{array}$ & $67(49.6)$ & $\begin{array}{l}5.51 \\
(3.69- \\
8.22)\end{array}$ & $\begin{array}{l}4.15 \\
(2.56- \\
6.73)\end{array}$ & $38(44.7)$ & $\begin{array}{l}4.20 \\
(2.59- \\
6.80)\end{array}$ & $\begin{array}{l}2.98 \\
(1.66- \\
5.34)\end{array}$ \\
\hline
\end{tabular}

Table 1. Odds ratios (OR) for any tinnitus, tinnitus as a big problem (TFI $\geq 48$ ) and severe tinnitus (THI $\geq 58$ ), and corresponding 95\% confidence intervals (CI), according to headache. TFI: Tinnitus Functional Index; THI: Tinnitus Handicap Inventory. *ORs were estimated using unconditional multiple logistic regression models after adjustment for sex, age, and level of education (model 1). Estimates in bold are statistically significant at 0.05 level. ${ }^{\circ}$ ORs were estimated using unconditional multiple logistic regression models after adjustment for sex, age, level of education, and hearing ability (model 2). Estimates in bold are statistically significant at 0.05 level. $\wedge$ Reference category.

increased neural activity in the auditory pathway ${ }^{28}$. Other structures such as the amygdala have been shown to interact with the auditory pathway in tinnitus patients ${ }^{29}$. Frontostriatal circuits, similar to those involved in chronic pain, have also been evidenced in tinnitus ${ }^{30}$. Auditory steady-state responses captured using magnetoencephalography reveal that the synchrony between different brain regions, such as the right parietal area and the anterior cingulate cortex correlates strongly with tinnitus intrusiveness ${ }^{31}$. However, headache and tinnitus also share common mechanisms characterized by changes in oscillatory activity called thalamocortical dysrhyth$\mathrm{mia}^{32-34}$. Furthermore, alterations in the trigeminal system form a basis for the development of headaches and migraines ${ }^{35,36}$ as well as for tinnitus ${ }^{16,37}$.

Using data from the Tinnitus Research Initiative (TRI) database, Langguth et al. evaluated the impact of self-reported headache in tinnitus patients and found that a larger proportion of those with headache complaints were female, with more frequent comorbid vertigo, temporomandibular joint complaints, neck pain and other pain disorders, and depressive symptoms ${ }^{18}$. Although a number of publications deal with the relationship between tinnitus and headache, only a limited number of these provide a measure of association between headaches and the risk of tinnitus after adjustment for potential confounding factors ${ }^{24,38,39}$. To our knowledge, none have addressed the risk with severe tinnitus. Here, we investigate this relationship in the Swedish Tinnitus Outreach Projet (STOP), a study designed to investigate the risk factors for tinnitus, and further analyze the phenotypic traits accompanying tinnitus when combined with headaches.

\section{Results}

Association study. We subjected STOP participants with $(\mathrm{n}=1,984)$ and without tinnitus $(\mathrm{n}=1,661)$ to the ESIT-SQ in order to evaluate the association between headaches and tinnitus. Within tinnitus cases, $13.3 \%$ reported having tinnitus as a big problem according to the TFI cut-off, and 7.9\% had severe tinnitus according to the THI cut-off. Subjects with headaches more frequently reported any tinnitus (multivariate OR, 2.61; 95\% $\mathrm{CI}, 2.19-3.12)$, tinnitus as a big problem (OR, 5.63; 95\% CI, 4.10-7.72) and severe tinnitus (OR, 4.99; 95\% CI, 3.41-7.32; Table 1). These associations persisted to a similar degree when considering the further adjustment for hearing ability (model 2, Table 1). We also evaluated the association between headache and tinnitus stratifying by sex. The relationship with any tinnitus persisted to the same degree in men (OR, 3.21; 95\% CI, 2.22-4.64) and in women (OR, 2.49; 95\% CI, 2.03-3.06). The association was greater in men, although not significantly different from women, both for tinnitus as a big problem (men: OR, 7.28; 95\% CI, 4.19-12.6 and women: OR, 5.51; 95\% CI, 3.69-8.22) and for severe tinnitus (men: OR, 8.14; 95\% CI, 4.22-15.7 and women: OR, 4.20; 95\% CI, 2.59-6.80). Thus, with increasing severity, the relationship between headache and tinnitus becomes stronger. 
Tinnitus phenotyping study. We next focused on tinnitus subjects only. Among 2,539 subjects with any tinnitus, $26 \%$ reported headache. This proportion increased for individuals reporting tinnitus as a big problem (36\%), and for those with severe tinnitus (40\%). Table 2 describes the sociodemographic characteristics of these three groups. There was a greater proportion of women in the any tinnitus groups with headaches in comparison to those without headaches. This gender bias survived the adjustment for multiple comparisons in the "any tinnitus" group but not in groups with more severe tinnitus. Any tinnitus subjects with headaches significantly differed from those without headaches in age, income, and employment status $(p=<0.0001)$ but not in marital status or education level. In contrast, in the samples with severe tinnitus, no differences were found in all sociodemographic variables after correction for multiple comparisons. We next used a set of questionnaires to assess the global impact of headache on tinnitus-related burden ${ }^{6,40}$. Subjects with any tinnitus who reported headache were found to have worse scores for all questionnaires $(p<0.0001$, Table 3$)$, with the exception of the numerical rating scale for awareness. In subjects with severe tinnitus, the only differences were found in the TFI, stress, anxiety measures, and physical and psychological quality of life ( $p=0.039$ to 0.025 after correction for multiple comparisons). Thus, headaches contribute to greater psychological burden in tinnitus subjects, regardless of the degree of severity.

When evaluating the tinnitus phenotypic characteristics for the group with any tinnitus, differences were observed in onset-related events $(p=0.006)$, with subjects more frequently reporting stress as the cause of their tinnitus (Table 4). Such effects were not seen in subjects with tinnitus as a big problem or severe tinnitus. Differences were also found in tinnitus loudness variation from day to day and sound of tinnitus $(p=0.001$ to 0.003$)$. Pulsatility was also different between headache and non-headache subjects with "any tinnitus" $(p<0.0001)$, although this could be due to a larger proportion in the headache group not being able to define it as pulsatile or not. Sounds also had a greater impact on subjects with any tinnitus and headaches, with greater worsening when exposed to loud noise, greater intolerance to sounds, and sounds causing pain and discomfort $(p=0.002$ to $<0.0001$, Tables 4 and 5$)$. The impact of stress, bad night sleep and naps also differed between any tinnitus subjects with or without headaches. A greater proportion of somatosensory impact such as tinnitus being affected by head movement and touch, self-reporting of temporomandibular complaints, vertigo and dizziness, neck pain and other pain syndromes was also found in any tinnitus subjects with headache $(p<0.0001)$. More subjects with headaches reported a diagnosed disease and were under psychiatric treatment $(p<0.0001)$, and a greater occurrence of tinnitus in the family was also reported in the headache group $(p=0.03)$. The proportion of subjects with self-reported hearing problems, hearing aids, and the location of tinnitus (laterality), did not differ between the headache and non-headache groups suggesting a minimal contribution of hearing loss into migraine in tinnitus subjects. Interestingly, in the severe tinnitus group, only vertigo/dizziness, neck pain and other pain syndromes remained different between the headache and non-headache groups ( $p=0.014$ to 0.002 , Table 5).

\section{Discussion}

Our study shows a strong relationship between headache and tinnitus with increasing severity. The relationship we found for any tinnitus $[\mathrm{OR}=2.19$ (95\% CI: 1.81-2.64)] is within the range of what has been reported for migraine in another cross-sectional study [multivariable-adjusted $\mathrm{OR}=1.77(1.36-2.30), \mathrm{n}=5729]^{24}$. The present work goes beyond this analysis by showing the increasing relationship between headache and tinnitus severity reaching an OR of $5.63(4.10-7.72)$ in the group with tinnitus as a big problem, suggesting either that headaches contribute to the distress caused by tinnitus, or that tinnitus distress contribute to headaches - a directionality that remains to be investigated.

Our study goes in line with findings from Langguth et al. ${ }^{18}$ as we replicate a number of points: vertigo/dizziness, neck pain and other pain syndromes constitute a dominant part of the symptomatology of subjects experiencing headaches, when having tinnitus as a big problem, or in severe tinnitus. A significant number of individuals with vertigo and migraine headache with tinnitus may fulfil diagnostic criteria for migraine ${ }^{41}$ or vestibular migraine ${ }^{42}$ and the association between tinnitus and migraine deserves further studies. Our study however included in a large part subjects from the population, rather than patients recruited in a clinical context, as those from the Tinnitus Research Initiative. As a consequence, a greater number of variables were found to differ between tinnitus subjects with or without headaches as we included a larger sample of the population. The any tinnitus group indeed includes subjects experiencing tinnitus occasionally (47\%) and permanently (53\%), where differences in income and employment could be found, in perception, psychological features (stress, anxiety and depression), fear of tinnitus, hyperacusis and quality of life. These aspects could not all be captured in the study by Langguth and colleagues (2017) most likely due to the fact that as severity increases, fewer differences are seen between the headache and non-headache tinnitus groups. Here, the prevalence of headache in tinnitus subjects started at $26 \%$ for the any tinnitus group, up to $40 \%$ in the severe tinnitus group as defined by the THI scale. These findings are consistent with the notion that headache, and particularly migraine, could be a facilitator to the development of severe tinnitus, as suggested with the greater risk in developing clinically significant tinnitus with migraines [adjusted Hazard Ratio: $3.30(2.17-5.00)]^{26}$ when compared to self-reported tinnitus of unknown severity [adjusted Recurrence Ratio: $1.28(1.06,1.56)]^{43}$.

In spite of the sample differences between STOP and the TRI database, the proportion of clinically significant tinnitus subjects with headache is higher in STOP (40\%) than in the TRI (27\%). Indeed, the county of Stockholm states in their regional guidelines a severe score based on the THI warrants a referral to specialist care ${ }^{44}$, which hence allows to infer that the severe tinnitus group presented here would be equivalent to clinically significant tinnitus. Differences between the two datasets could be either be regional or due to the fact that a broader range of troublesome tinnitus is included in the TRI. Unlike the Lannguth et al. study (2017), we were not able to perform the analysis according to the laterality of the headache, nor according to subtypes of headache (e.g. migraine, tension-type headache, cluster headache). There, painful sensations upon loud sound exposure were more frequent with bilateral headache, whereas cluster headache was the one most impacting on tinnitus distress. However, common to all types of headaches was the influence on comorbid vertigo, temporomandibular joint (TMJ) complaints, neck pain, and pain in general ${ }^{18}$, which are also observed in our study. 


\begin{tabular}{|c|c|c|c|c|c|c|}
\hline & \multicolumn{2}{|l|}{ Any Tinnitus } & \multicolumn{2}{|c|}{ Tinnitus as a big problem } & \multicolumn{2}{|c|}{ Severe tinnitus } \\
\hline & $\begin{array}{l}\text { Headache } \\
(n=660) \text { No }(\%)\end{array}$ & $\begin{array}{l}\text { No headache } \\
(\mathrm{n}=1879) \text { No }(\%)\end{array}$ & $\begin{array}{l}\text { Headache } \\
(\mathrm{n}=125) \\
\text { No }(\%)\end{array}$ & $\begin{array}{l}\text { No Headache } \\
(\mathrm{n}=218) \mathrm{n}(\%)\end{array}$ & $\begin{array}{l}\text { Headache } \\
(\mathrm{n}=79) \\
\text { No }(\%)\end{array}$ & $\begin{array}{l}\text { No Headache } \\
(\mathbf{n}=119) \mathbf{n}(\%)\end{array}$ \\
\hline Sex & \multicolumn{2}{|c|}{$\chi^{2}(1)=94.59, p<0.0001(<0.0001)$} & \multicolumn{2}{|c|}{$\begin{array}{l}\chi^{2}(1)=23.31, p<0.0001 \\
(p=0.0003)\end{array}$} & \multicolumn{2}{|c|}{$\begin{array}{l}\chi^{2}(1)=6.09, p=0.014 \\
(0.082)\end{array}$} \\
\hline Male & $225(34.1)$ & $1054(56.1)$ & $43(34.4)$ & $134(61.5)$ & $31(39.2)$ & $68(57.1)$ \\
\hline Female & $435(65.9)$ & $825(43.9)$ & $82(65.6)$ & $84(38.5)$ & $48(60.8)$ & $51(42.9)$ \\
\hline Age Group & \multicolumn{2}{|c|}{$\chi^{2}(7)=38.47, p<0.0001(<0.0001)$} & \multicolumn{2}{|c|}{$\begin{array}{l}\chi^{2}(7)=5.81, p=0.5619 \\
(0.5619)\end{array}$} & \multicolumn{2}{|c|}{$\begin{array}{l}\chi^{2}(7)=2.84, p=0.899 \\
(0.899)\end{array}$} \\
\hline$<24$ & $19(2.9)$ & $40(2.1)$ & $6(4.8)$ & $4(1.8)$ & $4(5.1)$ & $6(5.1)$ \\
\hline $25-34$ & $140(21.3)$ & $350(18.7)$ & $19(15.3)$ & $34(15.7)$ & $13(16.7)$ & $28(23.7)$ \\
\hline $35-44$ & $168(25.6)$ & $407(21.7)$ & $25(20.2)$ & $42(19.4)$ & $14(18)$ & $21(17.8)$ \\
\hline $45-54$ & $192(29.2)$ & $487(26)$ & $39(31.5)$ & $62(28.6)$ & $22(28.2)$ & $26(22)$ \\
\hline 55-64 & $88(13.4)$ & $272(14.5)$ & $20(16.1)$ & $37(17.1)$ & $14(18)$ & $21(17.8)$ \\
\hline $65-74$ & $43(6.5)$ & $268(14.3)$ & $12(9.7)$ & $24(11.1)$ & $9(11.5)$ & $11(9.3)$ \\
\hline $75-84$ & $6(0.9)$ & $48(2.6)$ & $2(1.6)$ & $12(5.5)$ & $2(2.6)$ & $4(3.4)$ \\
\hline$>85$ & $1(0.2)$ & $3(0.2)$ & $1(0.8)$ & $2(0.9)$ & $0(0)$ & $1(0.9)$ \\
\hline Marital Status & \multicolumn{2}{|c|}{$\chi^{2}(4)=7.84, p=0.0974(0.0974)$} & \multicolumn{2}{|c|}{$\begin{array}{l}\chi^{2}(4)=4.23, p=0.3757 \\
(0.45084)\end{array}$} & \multicolumn{2}{|c|}{$\begin{array}{l}\chi^{2}(4)=4.36, p=0.359 \\
(0.539)\end{array}$} \\
\hline Married & $268(40.6)$ & $830(44.2)$ & $40(32)$ & $91(41.7)$ & $29(36.7)$ & $43(36.1)$ \\
\hline Living with partner & $211(32)$ & $551(29.3)$ & $41(32.8)$ & $68(31.2)$ & $25(31.7)$ & $44(37)$ \\
\hline Single & $132(20)$ & $320(17)$ & $28(22.4)$ & $41(18.8)$ & $16(20.3)$ & $25(21)$ \\
\hline Widow/Widower & $5(0.8)$ & $26(1.4)$ & $3(2.4)$ & $3(1.4)$ & $1(1.3)$ & $3(2.5)$ \\
\hline Divorced & $44(6.7)$ & $152(8.1)$ & $13(10.4)$ & $15(6.9)$ & $8(10.1)$ & $4(3.4)$ \\
\hline Gross income & \multicolumn{2}{|c|}{$\chi^{2}(3)=37.56, p<0.0001(<0.0001)$} & \multicolumn{2}{|c|}{$\begin{array}{l}\chi^{2}(3)=12.72, p=0.0053 \\
(0.0159)\end{array}$} & \multicolumn{2}{|c|}{$\begin{array}{l}\chi^{2}(3)=5.08, p=0.166 \\
(0.332)\end{array}$} \\
\hline 0-200 000 SEK & $90(13.6)$ & $215(11.4)$ & $27(21.6)$ & $36(16.5)$ & $17(21.5)$ & $25(21)$ \\
\hline 200 001-450 000 SEK & $368(55.8)$ & $877(46.7)$ & $72(57.6)$ & $110(50.5)$ & $49(62)$ & $60(50.4)$ \\
\hline $450001 \mathrm{SEK}$ or more & $162(24.6)$ & $706(37.6)$ & $14(11.2)$ & $59(27.1)$ & $7(8.9)$ & $24(20.2)$ \\
\hline Don't know/don't want to disclose & $40(6.1)$ & $81(4.3)$ & $12(9.6)$ & $13(6)$ & $6(7.6)$ & $10(8.4)$ \\
\hline Education Level & \multicolumn{2}{|c|}{$\chi^{2}(3)=6.8, p=0.0785(0.0942)$} & \multicolumn{2}{|c|}{$\begin{array}{l}\chi^{2}(3)=5.1, p=0.1646 \\
(0.2469)\end{array}$} & \multicolumn{2}{|c|}{$\chi^{2}(3)=8, p=0.046(0.138)$} \\
\hline Middle School & $20(3)$ & $48(2.6)$ & $8(6.4)$ & $13(6)$ & $8(10.1)$ & $5(4.2)$ \\
\hline High School & $138(20.9)$ & $397(21.1)$ & $34(27.2)$ & $70(32.1)$ & $23(29.1)$ & $37(31.1)$ \\
\hline University & $424(64.2)$ & $1273(67.8)$ & $57(45.6)$ & $109(50)$ & $32(40.5)$ & 65 (54.6) \\
\hline Other & $78(11.8)$ & $161(8.6)$ & $26(20.8)$ & $26(11.9)$ & $16(20.3)$ & $12(10.1)$ \\
\hline Employment & \multicolumn{2}{|c|}{$\chi^{2}(10)=75.25, p<0.0001(<0.0001)$} & \multicolumn{2}{|c|}{$\begin{array}{l}\chi^{2}(8)=19.2, p=0.0138 \\
(0.0276)\end{array}$} & \multicolumn{2}{|c|}{$\begin{array}{l}\chi^{2}(7)=4.54, p=0.716 \\
(0.86)\end{array}$} \\
\hline Don't know & $2(0.3)$ & $0(0)$ & $0(0)$ & $0(0)$ & $0(0)$ & $0(0)$ \\
\hline Employed & $430(65.2)$ & $1140(60.7)$ & $59(47.2)$ & $111(50.9)$ & $39(49.4)$ & $59(49.6)$ \\
\hline Unemployed & $14(2.1)$ & $17(0.9)$ & $7(5.6)$ & $2(0.9)$ & $4(5.1)$ & $2(1.7)$ \\
\hline $\begin{array}{l}\text { Running my own business/ } \\
\text { Working as a partner in a company }\end{array}$ & $67(10.2)$ & $249(13.3)$ & $8(6.4)$ & $30(13.8)$ & $4(5.1)$ & $14(11.8)$ \\
\hline Retired & $44(6.7)$ & $286(15.2)$ & $15(12)$ & $37(17)$ & $11(13.9)$ & $14(11.8)$ \\
\hline $\begin{array}{l}\text { Sick leave (for more than two } \\
\text { month) or disability pension due to } \\
\text { illness or disability }\end{array}$ & $47(7.1)$ & $49(2.6)$ & $18(14.4)$ & $23(10.6)$ & $12(15.2)$ & $16(13.5)$ \\
\hline $\begin{array}{l}\text { Parental leave (since two months } \\
\text { or longer) }\end{array}$ & $13(2)$ & $27(1.4)$ & $3(2.4)$ & $3(1.4)$ & $1(1.3)$ & $2(1.7)$ \\
\hline Student & $28(4.2)$ & $79(4.2)$ & $10(8)$ & $10(4.6)$ & $7(8.9)$ & $10(8.4)$ \\
\hline Sabbatical & $3(0.5)$ & $2(0.1)$ & $1(0.8)$ & $0(0)$ & $0(0)$ & $0(0)$ \\
\hline Housewife/-Husband & $0(0)$ & $1(0.1)$ & $0(0)$ & $0(0)$ & $0(0)$ & $0(0)$ \\
\hline Other & $12(1.8)$ & $29(1.5)$ & $4(3.2)$ & $2(0.9)$ & $1(1.3)$ & $2(1.7)$ \\
\hline
\end{tabular}

Table 2. Sociodemographic and psychological characteristics for subjects with tinnitus with or without headache. Income refers to yearly income in SEK. Pairwise comparisons using Pearson's Chi-square test are reported. Percentages (\%) displayed refer to column percentages. $P$ values adjusted for multiple comparisons are shown between parenthesis. Estimates in bold are statistically significant at 0.05 level.

The frequent co-occurrence of pain symptoms in clinically significant tinnitus with comorbid TMJ complaints ${ }^{16,17}$ or headache ${ }^{18}$, is consistent with the hypothesis that tinnitus shares similar neurocircuitry to chronic pain, whereby frontostriatal gating would be affected ${ }^{30}$. Tinnitus could also emerge as a consequence of vestibular migraine, frequently observed in patients with episodic vertigo ${ }^{45,46}$. Additional mechanisms may occur such as 


\begin{tabular}{|c|c|c|c|c|c|c|}
\hline & \multicolumn{2}{|c|}{ Any Tinnitus } & \multicolumn{2}{|c|}{ Tinnitus as a big problem } & \multicolumn{2}{|c|}{ Severe tinnitus } \\
\hline & $\begin{array}{l}\text { Headache } \\
(n=660) \\
\text { mean }(S D)\end{array}$ & $\begin{array}{l}\text { No headache }(n=1879) \\
\text { mean }(S D)\end{array}$ & $\begin{array}{l}\text { Headache } \\
(\mathrm{n}=125) \\
\text { mean }(\mathrm{SD})\end{array}$ & $\begin{array}{l}\text { No headache } \\
(\mathrm{n}=218) \text { mean } \\
(\mathrm{SD})\end{array}$ & $\begin{array}{l}\text { Headache } \\
(n=79) \\
\text { mean (SD) }\end{array}$ & $\begin{array}{l}\text { No headache } \\
(n=119) \text { mean } \\
(\mathrm{SD})\end{array}$ \\
\hline \multirow[t]{2}{*}{ NRS Lo } & $44(25.5)$ & $39.5(25.3)$ & $72.4(19.3)$ & $70.6(19.1)$ & $76.1(18.4)$ & $72.8(20.0)$ \\
\hline & \multicolumn{2}{|c|}{$3.89, p=0.0001\left(0.0001,-0.10^{\dagger}\right)$} & \multicolumn{2}{|c|}{$0.87, p=0.3829\left(0.4248,-0.06^{\dagger}\right)$} & \multicolumn{2}{|c|}{$1.08, \mathrm{p}=0.2816\left(0.352,-0.09^{\dagger}\right)$} \\
\hline \multirow[t]{2}{*}{ NRS Aw } & $35.3(32.3)$ & $32.9(31.1)$ & $73.5(27.5)$ & $74(24.6)$ & $76.1(25.5)$ & $75(25.4)$ \\
\hline & \multicolumn{2}{|c|}{$1.87, \mathrm{p}=0.0612\left(0.0612,-0.05^{\dagger}\right)$} & \multicolumn{2}{|c|}{$0.36, \mathrm{p}=0.722\left(0.722,-0.02^{\dagger}\right)$} & \multicolumn{2}{|c|}{$1.08, \mathrm{p}=0.2816\left(0.352,-0.03^{\dagger}\right)$} \\
\hline \multirow[t]{2}{*}{ NRS An } & $23.3(27.4)$ & $19(24.8)$ & $62.4(28.0)$ & $58.9(26.2)$ & $68.7(27.2)$ & $64.4(26.9)$ \\
\hline & \multicolumn{2}{|c|}{$4.6, p<0.0001\left(<0.0001,-0.12^{\dagger}\right)$} & \multicolumn{2}{|c|}{$1.26, p=0.2059\left(0.2574,-0.08^{\dagger}\right)$} & \multicolumn{2}{|c|}{$1.29, \mathrm{p}=0.1978\left(0.2697,-0.11^{\dagger}\right)$} \\
\hline \multirow[t]{2}{*}{ THI } & $26.6(22.9)$ & $19.9(19.2)$ & $62.4(19.0)$ & $57.4(18.3)$ & $75.3(11.4)$ & $72(12)$ \\
\hline & \multicolumn{2}{|c|}{$7.09, \mathrm{p}<0.0001\left(<0.0001,-0.19^{\dagger}\right)$} & \multicolumn{2}{|c|}{$2.3, p=0.0212\left(0.0353,-0.15^{\dagger}\right)$} & \multicolumn{2}{|c|}{$2.15, p=0.0319\left(0.0684,-0.18^{\dagger}\right)$} \\
\hline \multirow[t]{2}{*}{ TFI } & $26.6(23.2)$ & $20.2(19.8)$ & $65.8(12.4)$ & $62.1(11.9)$ & $69.6(14.2)$ & $64.4(16.2)$ \\
\hline & \multicolumn{2}{|c|}{$6.07, \mathrm{p}<0.0001\left(<0.0001,-0.16^{\dagger}\right)$} & \multicolumn{2}{|c|}{$2.91, p=0.0036\left(0.0068,-0.18^{\dagger}\right)$} & \multicolumn{2}{|c|}{$2.48, p=0.0131\left(0.0393,-0.21^{\dagger}\right)$} \\
\hline \multirow[t]{2}{*}{ PSQ } & $0.4(0.2)$ & $0.3(0.2)$ & $0.5(0.2)$ & $0.5(0.2)$ & $0.6(0.2)$ & $0.5(0.2)$ \\
\hline & \multicolumn{2}{|c|}{$13.08, \mathrm{p}<0.0001\left(<0.0001,-0.34^{\dagger}\right)$} & \multicolumn{2}{|c|}{$4.54, \mathrm{p}<0.0001\left(0.0003,-0.29^{\dagger}\right)$} & \multicolumn{2}{|c|}{$2.94, p=0.0033\left(0.0248,-0.25^{\dagger}\right.$} \\
\hline \multirow[t]{2}{*}{ HADS_A } & $7.8(4.4)$ & $5.5(3.9)$ & $10.6(4.4)$ & $8.7(4.5)$ & $11.8(4.2)$ & $10.1(4.5)$ \\
\hline & \multicolumn{2}{|c|}{$11.59, \mathrm{p}<0.0001\left(<0.0001,-0.3^{\dagger}\right)$} & \multicolumn{2}{|c|}{$3.58, p=0.0003\left(0.0011,-0.23^{\dagger}\right)$} & $2.58, \mathrm{p}=0$ & $\left(0.0393,-0.22^{\dagger}\right)$ \\
\hline HADS_D & $4.7(3.7)$ & $3.3(3.2)$ & $7.6(3.9)$ & $6.4(3.9)$ & $8.3(4.0)$ & $7.5(4.1)$ \\
\hline & $9.15, p<C$ & $01\left(<0.0001,-0.24^{\dagger}\right)$ & $2.99, \mathrm{p}=0$ & $28\left(0.006,-0.19^{\dagger}\right)$ & $1.66, \mathrm{p}=\mathrm{c}$ & $2\left(0.1566,-0.14^{\dagger}\right)$ \\
\hline FTQ & $5.1(2.9)$ & $4.6(2.6)$ & $8.1(3.2)$ & $8.3(2.9)$ & $9(3.0)$ & $9.6(2.7)$ \\
\hline & $3.85, p=0.0$ & $01\left(0.0001,-0.1^{\dagger}\right)$ & $-0.85, \mathrm{p}=0$ & $965\left(0.4248,0.05^{\dagger}\right)$ & $-1.63, \mathrm{p}=0$ & $033\left(0.1566,0.14^{\dagger}\right)$ \\
\hline TCS & $14.9(11.5)$ & $11.7(9.8)$ & $28.1(11.0)$ & $26.9(9.4)$ & $31.4(9.6)$ & $30.4(8.7)$ \\
\hline & $6.02, \mathrm{p}<0$. & $1\left(<0.0001,-0.16^{\dagger}\right)$ & $1.53, \mathrm{p}=0.1$ & $9\left(0.1717,-0.1^{\dagger}\right)$ & $0.93, p=c$ & $2\left(0.4087,-0.08^{\dagger}\right)$ \\
\hline HQ & $20.2(9.3)$ & $15.5(8.9)$ & $26.3(7.7)$ & $23.3(8.8)$ & $27.3(8.0)$ & $25.5(8.8)$ \\
\hline & $11, \mathrm{p}<0.0$ & $\left(<0.0001,-0.29^{\dagger}\right)$ & $3.14, p=0$ & $17\left(0.0043,-0.2^{\dagger}\right)$ & $1.62, \mathrm{p}=0.1$ & $14\left(0.1566,-0.14^{\dagger}\right)$ \\
\hline QoL Phy & $14.4(2.9)$ & $16.1(2.5)$ & $12(2.7)$ & $13.7(2.7)$ & $11.6(2.8)$ & $13(2.7)$ \\
\hline & $-13.63, p$ & $.0001\left(<0.0001,0.36^{\dagger}\right)$ & $-5.29, \mathrm{p}<0$ & $001\left(0.0003,0.34^{\dagger}\right)$ & $-3.14, p=c$ & $017\left(0.0248,0.26^{\dagger}\right)$ \\
\hline QoL Psy & $13.9(2.9)$ & $15.2(2.5)$ & $11.8(2.9)$ & $13(2.8)$ & $11.1(2.8)$ & $12.3(2.8)$ \\
\hline & $-10.32, p$ & $.0001\left(<0.0001,0.27^{\dagger}\right)$ & $-3.55, p=0$ & $004\left(0.0012,0.23^{\dagger}\right)$ & $-2.49, \mathrm{p}=\mathrm{C}$ & $129\left(0.0393,0.21^{\dagger}\right)$ \\
\hline QoL Social & $13.6(3.2)$ & $14.4(3)$ & $12.2(3.6)$ & $13.2(3.3)$ & $12.3(3.2)$ & $12.7(3.6)$ \\
\hline & $-5.26, p<$ & $001\left(<0.0001,0.14^{\dagger}\right)$ & $-2.25, p=0$ & $248\left(0.0372,0.14^{\dagger}\right)$ & $-0.58, \mathrm{p}=0$ & $591\left(0.599,0.05^{\dagger}\right)$ \\
\hline QoL Env & $15.5(2.4)$ & $16.5(2.1)$ & $13.9(2.4)$ & $15(2.6)$ & $13.9(2.5)$ & $14.7(2.7)$ \\
\hline & $-9.81, p<$ & $01\left(<0.0001,0.25^{\dagger}\right)$ & $-3.99, \mathrm{p}<$ & $01\left(0.0003,0.26^{\dagger}\right)$ & $-2.25, \mathrm{p}=$ & $45\left(0.0613,0.19^{\dagger}\right)$ \\
\hline
\end{tabular}

Table 3. Questionnaire scores from subjects with tinnitus with or without headache. Values are mean $( \pm$ $\mathrm{SD})$. Pairwise comparisons using Wilcoxon's tests are reported below the compared values. P values adjusted for multiple comparisons are shown between parenthesis together with Cliff's $\delta$ (noted $\dagger$ ). Abbreviations: Numerical Ratings Score (NRS), Tinnitus loudness (Lo), Awareness (Aw), Annoyance (An), Tinnitus Handicap Inventory (THI), Tinnitus Functional Index (TFI), Fear of Tinnitus Questionnaire (FTQ), Tinnitus Catastrophising Scale (TCS), Hyperacusis Questionnaire (HQ), Perceived Stress Questionnaire (PSQ), Hospital Anxiety Depression Scales for Anxiety (HADS A) and depression (HADS D), Quality of Life (QoL) subscales from the World Health Organization: Physical (Phy), Psychological (Psych), Social (Soc), and Environmental (Env). Estimates in bold are statistically significant at 0.05 level.

an increased excitability of the trigeminal system ${ }^{47}$, also leading to TMJ and neck pain ${ }^{16}$. An increased occurrence of tinnitus in the family is observed with co-morbid headache, although this increase was not as large as what was found for TMJ complaints ${ }^{17}$. Recent evidence in twins and adoptees point towards a significant genetic contribution to tinnitus ${ }^{48,49}$. It is thus possible that the heritability of tinnitus shares common genetic mechanisms with that of pain and migraine. Indeed, genetics of migraine indicate a predisposition towards generalized neuronal excitability due to the inability to regulate glutamate availability ${ }^{19}$, as it has been recently suggested in a mouse model of tinnitus ${ }^{50}$. Recently, a genome-wide association studies (GWAS) of 375,000 individuals (including 59,674 cases) identified 38 susceptibility loci for migraine ${ }^{51}$. However, such large-scale studies in tinnitus are lacking and would be valuable to estimate the overlap between migraine and tinnitus ${ }^{52,53}$. However, while large biobanks with information on tinnitus do exist, the depth of the phenotyping is often limited which is why large biobanking efforts in ENT clinics have been encouraged ${ }^{54,55}$.

The phenotypic study on tinnitus-only subjects suggests a sex-bias, whereby the proportion of women with tinnitus increases from $44 \%$ to $66 \%$ in presence of headaches, as reported by Langguth et al. (2017). Similar sex-bias in women was also recently described in tinnitus with co-morbid TMJ complaints ${ }^{16,17}$. However, when non-tinnitus controls are included, the sex bias vanishes with instead a trend towards stronger relationship between tinnitus and headache in men, which emphasizes the need to include non-tinnitus controls to infer on sexual dimorphisms. The greater relationship in men did not significantly differ from that of women possibly due to the low sample size of the severe tinnitus groups, which warrants investigation in larger studies. Reasons 


\begin{tabular}{|c|c|c|c|c|c|c|}
\hline & \multicolumn{2}{|c|}{ Any Tinnitus } & \multicolumn{2}{|c|}{ Tinnitus as a big problem } & \multicolumn{2}{|c|}{ Severe tinnitus } \\
\hline & $\begin{array}{l}\text { Headache } \\
(\mathrm{n}=660) \\
\text { No }(\%)\end{array}$ & $\begin{array}{l}\text { No Headache } \\
(\mathrm{n}=1879) \text { No }(\%)\end{array}$ & $\begin{array}{l}\text { Headache } \\
(\mathrm{n}=125) \\
\text { No }(\%)\end{array}$ & $\begin{array}{l}\text { No Headache } \\
(n=218) \text { No }(\%)\end{array}$ & $\begin{array}{l}\text { Headache } \\
(\mathrm{n}=79) \\
\text { No }(\%)\end{array}$ & $\begin{array}{l}\text { No Headache } \\
(\mathrm{n}=119) \text { No }(\%)\end{array}$ \\
\hline Tinnitus onset & \multicolumn{2}{|c|}{$\chi^{2}(5)=5.02, p=0.414(0.497)$} & \multicolumn{2}{|c|}{$\chi^{2}(5)=1.31, p=0.934(0.946)$} & \multicolumn{2}{|c|}{$\chi^{2}(5)=3.91, p=0.562(0.919)$} \\
\hline Don’t know & $71(10.8)$ & $161(8.6)$ & $3(2.4)$ & $5(2.3)$ & $2(2.5)$ & $2(1.7)$ \\
\hline 0 to 6 months & $13(2.0)$ & $39(2.1)$ & $5(4.0)$ & $8(3.7)$ & $4(5.1)$ & $5(4.2)$ \\
\hline 6 months to 3 years & $105(15.9)$ & $265(14.1)$ & $17(13.6)$ & $37(17.0)$ & $14(17.7)$ & $24(20.2)$ \\
\hline 3 to 10 years & $183(27.7)$ & $546(29.1)$ & $32(25.6)$ & $61(28.0)$ & $21(26.6)$ & $31(26.1)$ \\
\hline 10 to 20 years & $180(27.3)$ & $559(29.8)$ & $39(31.2)$ & $64(29.4)$ & $18(22.8)$ & $38(31.9)$ \\
\hline More than 20 years & $108(16.4)$ & $309(16.4)$ & $29(23.2)$ & $43(19.7)$ & $20(25.3)$ & $19(16.0)$ \\
\hline Onset-related events & \multicolumn{2}{|c|}{$\chi^{2}(6)=19.68, p=0.003(0.006)$} & \multicolumn{2}{|c|}{$\chi^{2}(6)=8.52, p=0.203(0.405)$} & \multicolumn{2}{|c|}{$\chi^{2}(6)=2.26, p=0.894(0.919)$} \\
\hline Loud blast of sound & $224(33.9)$ & $759(40.4)$ & $35(28)$ & $71(32.6)$ & $23(29.1)$ & $33(27.7)$ \\
\hline Stress & $117(17.7)$ & $244(13)$ & $21(16.8)$ & $35(16.1)$ & $12(15.2)$ & $23(19.3)$ \\
\hline Change in hearing & $41(6.2)$ & $93(5.0)$ & $16(12.8)$ & $19(8.7)$ & $10(12.7)$ & $10(8.4)$ \\
\hline Head trauma & $6(0.9)$ & $15(0.8)$ & $0(0.0)$ & $2(0.9)$ & $0(0.0)$ & $1(0.8)$ \\
\hline Whiplash & $10(1.5)$ & $11(0.6)$ & $4(3.2)$ & $1(0.5)$ & $1(1.3)$ & $1(0.8)$ \\
\hline Other & $90(13.6)$ & $261(13.9)$ & $20(16)$ & $46(21.1)$ & $17(21.5)$ & $24(20.2)$ \\
\hline Don't know & $172(26.1)$ & $496(26.4)$ & $29(23.2)$ & $44(20.2)$ & $16(20.3)$ & $27(22.7)$ \\
\hline Tinnitus occurrence & \multicolumn{2}{|c|}{$\chi^{2}(1)=3.15, p=0.076(0.104)$} & \multicolumn{2}{|c|}{$\chi^{2}(1)=4.98, p=0.026(0.086)$} & \multicolumn{2}{|c|}{$\chi^{2}(1)=0.01, p=0.913(0.919)$} \\
\hline Occasionally (now and then) & $308(46.7)$ & $802(42.7)$ & $17(13.6)$ & $14(6.4)$ & $5(6.3)$ & $8(6.7)$ \\
\hline Always (all the time) & $352(53.3)$ & $1077(57.3)$ & $108(86.4)$ & 204 (93.6) & $74(93.7)$ & $111(93.3)$ \\
\hline $\begin{array}{l}\text { Time of the day of tinnitus } \\
\text { emergence }\end{array}$ & \multicolumn{2}{|c|}{$\chi^{2}(6)=13.8, p=0.032(0.05)$} & \multicolumn{2}{|c|}{$\chi^{2}(6)=4.71, p=0.581(0.758)$} & \multicolumn{2}{|c|}{$\chi^{2}(6)=3.05, p=0.802(0.919)$} \\
\hline Don’t know & $350(53)$ & $1012(53.9)$ & $51(40.8)$ & $85(39)$ & $28(35.4)$ & $35(29.4)$ \\
\hline When awakening & $34(5.2)$ & $123(6.6)$ & $14(11.2)$ & $31(14.2)$ & $13(16.5)$ & $22(18.5)$ \\
\hline In the morning & $12(1.8)$ & $35(1.9)$ & $6(4.8)$ & $5(2.3)$ & $3(3.8)$ & $4(3.4)$ \\
\hline Around noon & $37(5.6)$ & $125(6.7)$ & $12(9.6)$ & $24(11.0)$ & $9(11.4)$ & $16(13.5)$ \\
\hline In the afternoon & $31(4.7)$ & $93(5.0)$ & $7(5.6)$ & $19(8.7)$ & $6(7.6)$ & $16(13.5)$ \\
\hline In the evening & $71(10.8)$ & $238(12.7)$ & $21(16.8)$ & $38(17.4)$ & $15(19.0)$ & $17(14.3)$ \\
\hline Before sleeping & $125(18.9)$ & $253(13.5)$ & $14(11.2)$ & $16(7.3)$ & $5(6.3)$ & $9(7.6)$ \\
\hline Perceiving the onset of tinnitus & \multicolumn{2}{|c|}{$\chi^{2}(2)=6.12, p=0.047(0.067)$} & \multicolumn{2}{|c|}{$\chi^{2}(2)=0.59, p=0.744(0.88)$} & \multicolumn{2}{|c|}{$\chi^{2}(2)=0.23, p=0.891(0.919)$} \\
\hline Don’t know & $164(24.9)$ & $494(26.3)$ & $14(11.2)$ & $22(10.1)$ & $6(7.6)$ & $7(5.9)$ \\
\hline Gradual & $313(47.4)$ & $790(42.0)$ & $55(44.0)$ & $89(40.8)$ & $29(36.7)$ & $45(37.8)$ \\
\hline Abrupt & $183(27.7)$ & $595(31.7)$ & $56(44.8)$ & $107(49.1)$ & $44(55.7)$ & $67(56.3)$ \\
\hline Pulsatility & \multicolumn{2}{|c|}{$\begin{array}{l}\chi^{2}(3)=22.44, p<0.0001 \\
(0.0001)\end{array}$} & $x^{2}(3)=12$ & $08, p=0.007(0.03)$ & $x^{2}(3)=5.2$ & $1, \mathrm{p}=0.157(0.485)$ \\
\hline Don’t know & $77(11.7)$ & $138(7.3)$ & $20(16.0)$ & $22(10.1)$ & $5(6.3)$ & $14(11.8)$ \\
\hline Yes, with heart beat & $56(8.5)$ & $124(6.6)$ & $19(15.2)$ & $17(7.8)$ & $14(17.7)$ & $10(8.4)$ \\
\hline Yes, different from heart beat & $35(5.3)$ & $62(3.3)$ & $15(12.0)$ & $16(7.3)$ & $10(12.7)$ & $13(10.9)$ \\
\hline No & $492(74.6)$ & $1555(82.8)$ & $71(56.8)$ & $163(74.8)$ & $50(63.3)$ & $82(68.9)$ \\
\hline Location of tinnitus & $\chi^{2}(6)=13$. & $58, \mathrm{p}=0.035(0.052)$ & $x^{2}(6)=4.8$ & $4, p=0.565(0.758)$ & $x^{2}(6)=4.9$ & $7, \mathrm{p}=0.548(0.919)$ \\
\hline Right ear & $50(7.6)$ & $147(7.8)$ & $10(8.0)$ & $22(10.1)$ & $6(7.6)$ & $11(9.2)$ \\
\hline Left ear & $45(6.8)$ & $179(9.5)$ & $14(11.2)$ & $15(6.9)$ & $13(16.5)$ & $10(8.4)$ \\
\hline Both ears, worse in right & $141(21.4)$ & $305(16.2)$ & $24(19.2)$ & $35(16.1)$ & $13(16.5)$ & $24(20.2)$ \\
\hline Both ears, worse in left & $110(16.7)$ & $316(16.8)$ & $21(16.8)$ & $49(22.5)$ & $15(19)$ & $27(22.7)$ \\
\hline Both ears equally & $197(29.9)$ & $622(33.1)$ & $32(25.6)$ & $51(23.4)$ & $15(19)$ & $20(16.8)$ \\
\hline Inside the head & $108(16.4)$ & $289(15.4)$ & $19(15.2)$ & $40(18.4)$ & $12(15.2)$ & $23(19.3)$ \\
\hline Elsewhere & $9(1.4)$ & $21(1.1)$ & $5(4.0)$ & $6(2.8)$ & $5(6.3)$ & $4(3.4)$ \\
\hline Sound of tinnitus & $\chi^{2}(9)=27$ & $.04, p=0.001(0.003)$ & $\chi^{2}(7)=22$ & $48, p=0.002(0.013)$ & $\begin{array}{l}\chi^{2}(7)=14 \\
(0.204)\end{array}$ & $21, p=0.048$ \\
\hline Tone & $123(18.9)$ & $414(22.2)$ & $13(10.7)$ & $21(9.7)$ & $10(13)$ & $12(10.3)$ \\
\hline Noise & $65(10.0)$ & $217(11.6)$ & $9(7.4)$ & $26(12.0)$ & $7(9.1)$ & $9(7.7)$ \\
\hline Crickets & $18(2.8)$ & $71(3.8)$ & $2(1.6)$ & $7(3.2)$ & $2(2.6)$ & $5(4.3)$ \\
\hline Heartbeat & $5(0.8)$ & $6(0.3)$ & $1(0.8)$ & $1(0.5)$ & $2(2.6)$ & $0(0.0)$ \\
\hline Beeping & $67(10.3)$ & $233(12.5)$ & $6(4.9)$ & $34(15.7)$ & $4(5.2)$ & $17(14.5)$ \\
\hline Morse Code & $0(0.0)$ & $4(0.2)$ & $0(0.0)$ & $0(0.0)$ & $0(0.0)$ & $0(0.0)$ \\
\hline An alarm & $9(1.4)$ & $11(0.6)$ & $4(3.3)$ & $2(0.9)$ & $3(3.9)$ & $2(1.7)$ \\
\hline Other & $11(1.7)$ & $62(3.3)$ & $1(0.8)$ & $13(6)$ & $1(1.3)$ & $11(9.4)$ \\
\hline
\end{tabular}




\begin{tabular}{|c|c|c|c|c|c|c|}
\hline & \multicolumn{2}{|c|}{ Any Tinnitus } & \multicolumn{2}{|c|}{ Tinnitus as a big problem } & \multicolumn{2}{|c|}{ Severe tinnitus } \\
\hline & \begin{tabular}{l|} 
Headache \\
$(\mathrm{n}=660)$ \\
No $(\%)$
\end{tabular} & $\begin{array}{l}\text { No Headache } \\
(\mathbf{n}=1879) \text { No }(\%)\end{array}$ & $\begin{array}{l}\text { Headache } \\
(\mathrm{n}=125) \\
\text { No }(\%)\end{array}$ & $\begin{array}{l}\text { No Headache } \\
(\mathrm{n}=218) \text { No }(\%)\end{array}$ & $\begin{array}{l}\text { Headache } \\
(\mathrm{n}=79) \\
\text { No }(\%)\end{array}$ & $\begin{array}{l}\text { No Headache } \\
(n=119) \text { No }(\%)\end{array}$ \\
\hline Don't know & $1(0.2)$ & $1(0.1)$ & $0(0)$ & $0(0.0)$ & $0(0.0)$ & $0(0.0)$ \\
\hline Complex & $352(54.1)$ & $848(45.4)$ & $86(70.5)$ & $112(51.9)$ & $48(62.3)$ & $61(52.1)$ \\
\hline $\begin{array}{l}\text { Tinnitus loudness variation } \\
\text { from day to day }\end{array}$ & \multicolumn{2}{|c|}{$\chi^{2}(5)=23.14, p=0(0.001)$} & \multicolumn{2}{|c|}{$\chi^{2}(5)=3.53, p=0.62(0.775)$} & \multicolumn{2}{|c|}{$\chi^{2}(5)=4.41, p=0.493(0.919)$} \\
\hline Don't know & $36(5.5)$ & $127(6.8)$ & $4(3.2)$ & $6(2.8)$ & $1(1.3)$ & $2(1.7)$ \\
\hline Never & $38(5.8)$ & $154(8.2)$ & $10(8.0)$ & $18(8.3)$ & $6(7.6)$ & $6(5.0)$ \\
\hline Seldom & $79(12.0)$ & $301(16.0)$ & $19(15.2)$ & $35(16.1)$ & $11(13.9)$ & $14(11.8)$ \\
\hline Sometimes & $241(36.5)$ & $710(37.8)$ & $45(36.0)$ & $94(43.1)$ & $27(34.2)$ & $54(45.4)$ \\
\hline Often & $186(28.2)$ & $397(21.1)$ & $30(24.0)$ & 47 (21.6) & $19(24.1)$ & $30(25.2)$ \\
\hline Always & $80(12.1)$ & $190(10.1)$ & $17(13.6)$ & $18(8.3)$ & $15(19)$ & $13(10.9)$ \\
\hline Pitch of tinnitus & \multicolumn{2}{|c|}{$\chi^{2}(4)=0.12, p=0.998(0.998)$} & \multicolumn{2}{|c|}{$\chi^{2}(4)=1.74, p=0.783(0.88)$} & \multicolumn{2}{|c|}{$\chi^{2}(4)=2.55, p=0.636(0.919)$} \\
\hline Don't know & $27(4.1)$ & $72(3.8)$ & $4(3.2)$ & $5(2.3)$ & $2(2.5)$ & $3(2.5)$ \\
\hline Very high frequency & $157(23.8)$ & $450(24.0)$ & $39(31.2)$ & $64(29.4)$ & $30(38.0)$ & $38(31.9)$ \\
\hline High frequency & $293(44.4)$ & $838(44.6)$ & $54(43.2)$ & $101(46.3)$ & $30(38.0)$ & 47 (39.5) \\
\hline Medium frequency & $128(19.4)$ & $366(19.5)$ & $24(19.2)$ & $36(16.5)$ & $14(17.7)$ & $20(16.8)$ \\
\hline Low frequency & $55(8.3)$ & $153(8.1)$ & $4(3.2)$ & $12(5.5)$ & $3(3.8)$ & $11(9.2)$ \\
\hline $\begin{array}{l}\text { Reduction of tinnitus by music } \\
\text { or environmental sounds }\end{array}$ & \multicolumn{2}{|c|}{$\chi^{2}(2)=0.48, p=0.787(0.827)$} & \multicolumn{2}{|c|}{$\chi^{2}(2)=2.02, p=0.364(0.682)$} & \multicolumn{2}{|c|}{$\chi^{2}(2)=6.16, p=0.046(0.204)$} \\
\hline Don’t know & $171(25.9)$ & $470(25.0)$ & $23(18.4)$ & $29(13.3)$ & $15(19)$ & $10(8.4)$ \\
\hline Yes & $367(55.6)$ & $1074(57.2)$ & $68(54.4)$ & $133(61.0)$ & $45(57.0)$ & $67(56.3)$ \\
\hline No & $122(18.5)$ & $335(17.8)$ & $34(27.2)$ & $56(25.7)$ & $19(24.1)$ & $42(35.3)$ \\
\hline $\begin{array}{l}\text { Worsening of tinnitus by loud } \\
\text { noise }\end{array}$ & \multicolumn{2}{|c|}{$\chi^{2}(2)=14.57, p=0.001(0.002)$} & \multicolumn{2}{|c|}{$\chi^{2}(2)=1.36, p=0.506(0.758)$} & \multicolumn{2}{|c|}{$\chi^{2}(2)=0.7, p=0.703(0.919)$} \\
\hline Don't know & $181(27.4)$ & $525(27.9)$ & $30(24.0)$ & 47 (21.6) & $21(26.6)$ & 27 (22.7) \\
\hline Yes & $357(54.1)$ & $881(46.9)$ & 77 (61.6) & $129(59.2)$ & 47 (59.5) & $71(59.7)$ \\
\hline No & $122(18.5)$ & $473(25.2)$ & $18(14.4)$ & $42(19.3)$ & $11(13.9)$ & $21(17.7)$ \\
\hline $\begin{array}{l}\text { Tinnitus affected by head } \\
\text { movement or touch }\end{array}$ & \multicolumn{2}{|c|}{$\begin{array}{l}\chi^{2}(2)=38.19, p<0.0001 \\
(0.0001)\end{array}$} & \multicolumn{2}{|c|}{$\chi^{2}(2)=6.91, p=0.032(0.095)$} & \multicolumn{2}{|c|}{$\chi^{2}(2)=0.8, p=0.67(0.919)$} \\
\hline Don't know & $157(23.8)$ & $361(19.2)$ & $15(12.0)$ & $35(16.1)$ & $8(10.1)$ & $12(10.1)$ \\
\hline Yes & $210(31.8)$ & $426(22.7)$ & $61(48.8)$ & $75(34.4)$ & $42(53.2)$ & $56(47.1)$ \\
\hline No & $293(44.4)$ & $1092(58.1)$ & $49(39.2)$ & $108(49.5)$ & $29(36.7)$ & $51(42.9)$ \\
\hline Tinnitus affected by nap & \multicolumn{2}{|c|}{$\chi^{2}(3)=9.61, p=0.022(0.037)$} & $\chi^{2}(3)=1.9$ & $9, \mathrm{p}=0.574(0.758)$ & $x^{2}(3)=0.5$ & $\mathrm{p}=0.919(0.919)$ \\
\hline Don't know & $373(56.5)$ & $1068(56.8)$ & $43(34.4)$ & 88 (40.4) & $25(31.7)$ & $42(35.3)$ \\
\hline It mainly worsens my tinnitus & $15(2.3)$ & $38(2.0)$ & $7(5.6)$ & $16(7.3)$ & $8(10.1)$ & $12(10.1)$ \\
\hline It mainly reduces my tinnitus & $199(30.2)$ & $633(33.7)$ & $62(49.6)$ & $93(42.7)$ & 39 (49.4) & $53(44.5)$ \\
\hline It has no effect & $73(11.1)$ & $140(7.5)$ & $13(10.4)$ & $21(9.6)$ & $7(8.9)$ & $12(10.1)$ \\
\hline $\begin{array}{l}\text { Tinnitus affected by bad nights } \\
\text { sleep }\end{array}$ & $\begin{array}{l}\chi^{2}(5)=51 . \\
(0.0001)\end{array}$ & $73, p<0.0001$ & $x^{2}(5)=7.6$ & $2, \mathrm{p}=0.179(0.383)$ & $\chi^{2}(5)=6.2$ & $9, \mathrm{p}=0.279(0.76)$ \\
\hline Don't know & $257(38.9)$ & 704 (37.5) & $25(20)$ & $42(19.3)$ & $14(17.7)$ & $15(12.6)$ \\
\hline Never & $53(8)$ & $315(16.8)$ & $6(4.8)$ & $24(11.0)$ & $3(3.8)$ & $10(8.4)$ \\
\hline Seldom & $43(6.5)$ & $154(8.2)$ & $9(7.2)$ & $20(9.2)$ & $6(7.6)$ & $12(10.1)$ \\
\hline Sometimes & $145(22)$ & $419(22.3)$ & $28(22.4)$ & $58(26.6)$ & $14(17.7)$ & $33(27.7)$ \\
\hline Often & $122(18.5)$ & $221(11.8)$ & $39(31.2)$ & $54(24.8)$ & $27(34.2)$ & $33(27.7)$ \\
\hline Always & $40(6.1)$ & $66(3.5)$ & $18(14.4)$ & $20(9.2)$ & $15(19)$ & $16(13.5)$ \\
\hline Tinnitus affected by stress & $\chi^{2}(3)=69$. & $4, \mathrm{p}<0.0001(0.0001)$ & $\chi^{2}(2)=7.5$ & $\mathrm{p}=0.024(0.086)$ & $\chi^{2}(2)=1.4$ & $3, \mathrm{p}=0.491(0.919)$ \\
\hline Don't know & $211(32)$ & $677(36)$ & $23(18.4)$ & $50(22.9)$ & $12(15.2)$ & $20(16.8)$ \\
\hline Yes, it worsens my tinnitus & $369(55.9)$ & $738(39.3)$ & $90(72)$ & $127(58.3)$ & $62(78.5)$ & $86(72.3)$ \\
\hline Yes, it reduces my tinnitus & $79(12)$ & $460(24.5)$ & $12(9.6)$ & $41(18.8)$ & $5(6.3)$ & $13(10.9)$ \\
\hline No, it has no effect & $1(0.2)$ & $4(0.2)$ & $0(0.0)$ & $0(0.0)$ & $0(0.0)$ & $0(0.0)$ \\
\hline Tinnitus affected by medication & $\chi^{2}(2)=0.95$ & $5, \mathrm{p}=0.623(0.692)$ & $\chi^{2}(2)=0.1$ & $1, p=0.946(0.946)$ & $\chi^{2}(2)=0.3$ & $2, \mathrm{p}=0.854(0.919)$ \\
\hline Don’t know & $482(73)$ & $1380(73.4)$ & $83(66.4)$ & $141(64.7)$ & $46(58.2)$ & $74(62.2)$ \\
\hline Yes & $20(3.0)$ & $44(2.3)$ & $9(7.2)$ & $16(7.3)$ & $9(11.4)$ & $12(10.1)$ \\
\hline No & $158(23.9)$ & $455(24.2)$ & $33(26.4)$ & $61(28.0)$ & $24(30.4)$ & $33(27.7)$ \\
\hline $\begin{array}{l}\text { Contacted a clinician due to } \\
\text { tinnitus }\end{array}$ & $\chi^{2}(2)=1.14$ & $4, p=0.566(0.653)$ & $x^{2}(2)=1$ & $8, p=0.391(0.69)$ & $\chi^{2}(2)=0.1$ & $8, p=0.916(0.919)$ \\
\hline No & $415(62.9)$ & $1209(64.3)$ & $32(25.6)$ & $43(19.7)$ & $11(13.9)$ & $17(14.3)$ \\
\hline Continued & & & & & & \\
\hline
\end{tabular}




\begin{tabular}{|c|c|c|c|c|c|c|}
\hline & \multicolumn{2}{|c|}{ Any Tinnitus } & \multicolumn{2}{|c|}{ Tinnitus as a big problem } & \multicolumn{2}{|c|}{ Severe tinnitus } \\
\hline & $\begin{array}{l}\text { Headache } \\
(\mathrm{n}=660) \\
\text { No }(\%)\end{array}$ & $\begin{array}{l}\text { No Headache } \\
(\mathrm{n}=1879) \text { No }(\%)\end{array}$ & $\begin{array}{l}\text { Headache } \\
(\mathrm{n}=125) \\
\text { No }(\%)\end{array}$ & $\begin{array}{l}\text { No Headache } \\
(\mathrm{n}=218) \text { No }(\%)\end{array}$ & $\begin{array}{l}\text { Headache } \\
(\mathbf{n}=79) \\
\text { No }(\%)\end{array}$ & $\begin{array}{l}\text { No Headache } \\
(\mathrm{n}=119) \text { No }(\%)\end{array}$ \\
\hline Yes, because of curiosity & $32(4.9)$ & $102(5.4)$ & $3(2.4)$ & $8(3.7)$ & $2(2.5)$ & $2(1.7)$ \\
\hline Yes, because I sought for help & $213(32.3)$ & $568(30.2)$ & $90(72.0)$ & $167(76.6)$ & $66(83.5)$ & $100(84.0)$ \\
\hline Number of tinnitus treatments & \multicolumn{2}{|c|}{$\chi^{2}(3)=3.18, p=0.365(0.456)$} & \multicolumn{2}{|c|}{$\mathrm{X}^{2}(3)=0.83, \mathrm{p}=0.842(0.902)$} & \multicolumn{2}{|c|}{$\chi^{2}(3)=2.29, p=0.514(0.919)$} \\
\hline None & $563(85.3)$ & $1651(87.9)$ & $80(64)$ & $134(61.5)$ & $42(53.2)$ & $61(51.3)$ \\
\hline 1 & $41(6.2)$ & $96(5.1)$ & $17(13.6)$ & $30(13.8)$ & $14(17.7)$ & $17(14.3)$ \\
\hline $2-4$ & $42(6.4)$ & $94(5.0)$ & $19(15.2)$ & $32(14.7)$ & $16(20.3)$ & $22(18.5)$ \\
\hline 5 or more & $14(2.1)$ & $38(2.0)$ & $9(7.2)$ & $22(10.1)$ & $7(8.9)$ & $19(16)$ \\
\hline Tinnitus occurence in family & \multicolumn{2}{|c|}{$\chi^{2}(1)=5.64, p=0.018(0.031)$} & \multicolumn{2}{|c|}{$\chi^{2}(1)=0.52, p=0.471(0.744)$} & \multicolumn{2}{|c|}{$\chi^{2}(1)=0.05, p=0.832(0.919)$} \\
\hline No & $518(78.5)$ & $1553(82.7)$ & $89(71.2)$ & $163(74.8)$ & $56(70.9)$ & $86(72.3)$ \\
\hline Yes & $142(21.5)$ & $326(17.4)$ & $36(28.8)$ & $55(25.2)$ & $23(29.1)$ & $33(27.7)$ \\
\hline
\end{tabular}

Table 4. Phenotypic characteristics for subjects with tinnitus with or without headache. Pairwise comparisons using Pearson's Chi-square test are reported. Percentages (\%) displayed refer to column percentages. P values adjusted for multiple comparisons are shown between parenthesis. Estimates in bold are statistically significant at 0.05 level.

for the increased proportion of women with tinnitus when reporting headache could also be due to the fact that migraine and TMJ disorders are more frequent in women (3:1 female to male ratio in migraine; and 2:1 in TMJ disorders $)^{56,57}$. Headaches are indeed among the leading causes of years lived with disability in Scandinavian women $^{58}$. It is interesting to note though that greater stress due to tinnitus has been reported in women ${ }^{6,59}$ and that a greater association of tinnitus with suicide attempts was found in women but not in men $^{7}$, underlying that the greater psychological impact of tinnitus in women should not be disregarded. Our work encourages the need of including sex as a biological variable (SABV) in future tinnitus studies.

There are a number of limitations to our study, which include self-reported tinnitus and headaches. Both of them are complex and heterogeneous conditions that pre-empt clinical examination. Indeed, our study incorporates participants that may not be representative of the clinical setting. Self-reports may yield different prevalence estimates than diagnostic codes from medical records, the latter representing more a severe form of tinnitus for which medical care is sought. Reliance on self-report introduces the possibility of recall bias, that would be otherwise minimized when involving a physician. However, as regional guidelines recommend a referral to specialty care when a severe THI score is reached ${ }^{44}$, one could - at least in part - infer on the phenotypic characteristics that would be expected in the clinic. Secondly, while STOP participants were recruited from LifeGene, which is representative of the general population, it is unclear whether our findings can be generalized to that of the general population. Not all LifeGene participants with tinnitus joined STOP, and as a consequence, a recruitment bias may have occurred e.g. towards more participants seeking treatment options. Thirdly, as headaches and tinnitus were assessed at one single point in time, it is not possible to understand the direction of the observed relationship.

Overall, our study confirms that tinnitus with co-morbid headaches is accompanied with vertigo, neck and other pain syndromes. The association between headache and tinnitus increases as tinnitus becomes more severe. Thus, an interprofessional approach to care would be warranted in these cases. Future longitudinal studies investigating the conjunct impact of headache with TMJ disorders may offer additional insights into their additive or synergistic contribution to tinnitus.

\section{Materials and Methods}

Sample. Participants were invited to join the Swedish Tinnitus Outreach Project via social media channels and through partnerships with local cohorts, including LifeGene ${ }^{60}$. All participants above 18 years of age were eligible. Voluntary registration was done on a website from STOP (https://stop.ki.se). After providing informed consent for having their data stored in a database and analyzed, participants were invited to fill an online questionnaire, which was answered between November 2015 and January 2018. The first part of this study was performed on a subset of individuals with or without tinnitus who responded to the European School for Interdisciplinary Tinnitus Research - Screening Questionnaire (ESIT-SQ) to investigate the association between headaches and tinnitus. The second part of this study focused only on tinnitus subjects with or without headaches in order to investigate their phenotypic traits. We followed the STROBE guidelines and the checklist is available in the online materials. All subjects gave written informed consent in accordance with the Declaration of Helsinki. The project has been approved by the Regional Ethics Review Board in Stockholm (2015/2129-31/1).

Association study. The ESIT-SQ was designed to be comprehensive with a specific attention to risk factors that could be answered by people with or without tinnitus ${ }^{61}$. Here the tinnitus question A17 was: "Tinnitus refers to the perception of noise in your head or ears (such as ringing or buzzing) in the absence of any corresponding source of sound external to your head. Over the past year, have you had tinnitus in your head or in one or both ears that lasts for more than five minutes at a time?" with answer options being: "do not know", "no never", "no, not in the past year", "yes, some of the time", "yes a lot of time", "yes most of the time". Those answering "don't know" or "no, not in the past year" were excluded from the analysis. Question A15 stated "Do you suffer from any of the following pain syndromes?" of which Headache was one of the options. For the present analysis, we thus included 1984 tinnitus cases and 1661 subjects without tinnitus from the STOP project. 


\begin{tabular}{|c|c|c|c|c|c|c|}
\hline \multirow{4}{*}{\begin{tabular}{|l|} 
\\
Hearing problem \\
Don't know \\
\end{tabular}} & \multicolumn{2}{|l|}{ Any Tinnitus } & \multicolumn{2}{|c|}{ Tinnitus as a big problem } & \multicolumn{2}{|c|}{ Severe tinnitus } \\
\hline & \begin{tabular}{|l|} 
Headache \\
$(\mathrm{n}=660)$ No \\
$(\%)$
\end{tabular} & $\begin{array}{l}\begin{array}{l}\text { No Headache } \\
(\mathrm{n}=1879) \text { No } \\
(\%)\end{array} \\
\end{array}$ & $\begin{array}{l}\text { Headache } \\
(n=125) \text { No } \\
(\%)\end{array}$ & $\begin{array}{l}\text { No Headache } \\
(\mathrm{n}=218) \text { No } \\
(\%)\end{array}$ & $\begin{array}{l}\text { Headache } \\
(\mathrm{n}=79) \text { No } \\
(\%)\end{array}$ & $\begin{array}{l}\text { No Headache } \\
(\mathrm{n}=119) \text { No } \\
(\%)\end{array}$ \\
\hline & \multicolumn{2}{|c|}{$\chi^{2}(2)=2.86, p=0.24(0.313)$} & \multicolumn{2}{|c|}{$\chi^{2}(2)=1.57, p=0.456(0.744)$} & \multicolumn{2}{|c|}{$\chi^{2}(2)=0.36, p=0.836(0.919)$} \\
\hline & $121(18.3)$ & $304(16.2)$ & $14(11.2)$ & $27(12.4)$ & $6(7.6)$ & $12(10.1)$ \\
\hline Yes & $309(46.8)$ & $946(50.4)$ & $81(64.8)$ & $151(69.3)$ & $50(63.3)$ & $73(61.3)$ \\
\hline No & $230(34.9)$ & $629(33.5)$ & $30(24)$ & 40 (18.4) & $23(29.1)$ & $34(28.6)$ \\
\hline Hearing aids & \multicolumn{2}{|c|}{$\chi^{2}(3)=1.01, p=0.799(0.827)$} & \multicolumn{2}{|c|}{$\chi^{2}(3)=1.04, p=0.792(0.88)$} & \multicolumn{2}{|c|}{$\chi^{2}(3)=1.2, p=0.754(0.919)$} \\
\hline Yes, on both ears & $43(6.5)$ & $115(6.1)$ & $22(17.6)$ & $43(19.7)$ & $12(15.2)$ & $22(18.5)$ \\
\hline Yes, on the right ear & $4(0.6)$ & $19(1.0)$ & $4(3.2)$ & $6(2.8)$ & $3(3.8)$ & $4(3.4)$ \\
\hline Yes, on the left ear & $8(1.2)$ & $23(1.2)$ & $5(4.0)$ & $5(2.3)$ & $4(5.1)$ & $3(2.5)$ \\
\hline No & $605(91.7)$ & $1722(91.6)$ & $94(75.2)$ & $164(75.2)$ & $60(76.0)$ & $90(75.6)$ \\
\hline Problems tolerating sounds & \multicolumn{2}{|c|}{$\begin{array}{l}\chi^{2}(4)=70.08, p<0.0001 \\
(0.0001)\end{array}$} & \multicolumn{2}{|c|}{$\chi^{2}(4)=9.81, p=0.044(0.119)$} & \multicolumn{2}{|c|}{$\chi^{2}(3)=5.15, p=0.162(0.485)$} \\
\hline Never & $11(1.7)$ & $95(5.1)$ & $0(0.0)$ & $1(0.5)$ & $0(0.0)$ & $0(0.0)$ \\
\hline Rarely & $61(9.2)$ & $347(18.5)$ & $4(3.2)$ & $16(7.3)$ & $2(2.5)$ & $9(7.6)$ \\
\hline Sometimes & $248(37.6)$ & $742(39.5)$ & $24(19.2)$ & $66(30.3)$ & $14(17.7)$ & $31(26.1)$ \\
\hline Usually & $207(31.4)$ & $469(25)$ & $49(39.2)$ & $73(33.5)$ & $28(35.4)$ & $39(32.8)$ \\
\hline Always & $133(20.2)$ & $226(12)$ & $48(38.4)$ & $62(28.4)$ & $35(44.3)$ & $40(33.6)$ \\
\hline $\begin{array}{l}\text { Sounds cause pain or physical } \\
\text { discomfort }\end{array}$ & \multicolumn{2}{|c|}{$\begin{array}{l}\chi^{2}(2)=51.96, p<0.0001 \\
(0.0001)\end{array}$} & \multicolumn{2}{|c|}{$\chi^{2}(2)=6, p=0.05(0.124)$} & \multicolumn{2}{|c|}{$\chi^{2}(2)=0.69, p=0.71(0.919)$} \\
\hline Don’t know & $39(5.9)$ & $115(6.1)$ & $7(5.6)$ & $16(7.3)$ & $3(3.8)$ & $7(5.9)$ \\
\hline Yes & $427(64.7)$ & $919(48.9)$ & $98(78.4)$ & $144(66.1)$ & $62(78.5)$ & $88(74)$ \\
\hline No & $194(29.4)$ & $845(45)$ & $20(16)$ & $58(26.6)$ & $14(17.7)$ & $24(20.2)$ \\
\hline Temporomandibular problems & \multicolumn{2}{|c|}{$\begin{array}{l}\chi^{2}(2)=168.49, p<0.0001 \\
(0.0001)\end{array}$} & \multicolumn{2}{|c|}{$\chi^{2}(2)=15.98, p=0(0.002)$} & \multicolumn{2}{|c|}{$\chi^{2}(2)=9.15, p=0.01(0.077)$} \\
\hline Don't know & $37(5.6)$ & $64(3.4)$ & $11(8.8)$ & $17(7.8)$ & $6(7.6)$ & $7(5.9)$ \\
\hline Yes & $231(35)$ & $245(13)$ & $50(40)$ & $45(20.6)$ & $36(45.6)$ & $31(26.1)$ \\
\hline No & $392(59.4)$ & $1570(83.6)$ & $64(51.2)$ & $156(71.6)$ & $37(46.8)$ & $81(68.1)$ \\
\hline Vertigo/dizziness & \multicolumn{2}{|c|}{$\begin{array}{l}\chi^{2}(2)=177.98, p<0.0001 \\
(0.0001)\end{array}$} & \multicolumn{2}{|c|}{$\begin{array}{l}\chi^{2}(2)=38.25, p<0.0001 \\
(0.0005)\end{array}$} & \multicolumn{2}{|c|}{$\begin{array}{l}\chi^{2}(2)=24.39, p<0.0001 \\
(0.002)\end{array}$} \\
\hline Don't know & $33(5.0)$ & $64(3.4)$ & $6(4.8)$ & $9(4.1)$ & $1(1.3)$ & $4(3.4)$ \\
\hline Yes & $278(42.1)$ & $324(17.2)$ & $67(53.6)$ & $47(21.6)$ & $40(50.6)$ & $21(17.7)$ \\
\hline No & $349(52.9)$ & $1491(79.4)$ & $52(41.6)$ & $162(74.3)$ & $38(48.1)$ & $94(79.0)$ \\
\hline Neck pain & \multicolumn{2}{|c|}{$\begin{array}{l}\chi^{2}(2)=339.35, p<0.0001 \\
(0.0001)\end{array}$} & \multicolumn{2}{|c|}{$\begin{array}{l}\chi^{2}(2)=36.22, p<0.0001 \\
(0.0005)\end{array}$} & $\begin{array}{l}\chi^{2}(2)=13 \\
(0.014)\end{array}$ & $p=0.001$ \\
\hline Don't know & $14(2.1)$ & $26(1.4)$ & $3(2.4)$ & $6(2.8)$ & $1(1.3)$ & $1(0.8)$ \\
\hline Yes & $397(60.2)$ & $410(21.8)$ & $83(66.4)$ & $72(33.0)$ & $51(64.6)$ & $46(38.7)$ \\
\hline No & $249(37.7)$ & $1443(76.8)$ & $39(31.2)$ & $140(64.2)$ & $27(34.2)$ & $72(60.5)$ \\
\hline Other pain syndromes & $\begin{array}{l}\chi^{2}(2)=145.1 \\
(0.0001)\end{array}$ & $\mathrm{p}<0.0001$ & $\begin{array}{l}\chi^{2}(2)=33 . \\
(0.0005)\end{array}$ & $\mathrm{p}<0.0001$ & $\chi^{2}(2)=17$ & $=0(0.003)$ \\
\hline Don't know & $24(3.6)$ & $18(1.0)$ & $4(3.2)$ & $2(0.9)$ & $3(3.8)$ & $3(2.5)$ \\
\hline Yes & $256(38.8)$ & $341(18.2)$ & $71(56.8)$ & $60(27.5)$ & $43(54.4)$ & $31(26.1)$ \\
\hline No & $380(57.6)$ & $1520(80.9)$ & $50(40)$ & $156(71.6)$ & $33(41.8)$ & $85(71.4)$ \\
\hline Under psychatric treatment & $\begin{array}{l}\chi^{2}(2)=34.4 \\
(0.0001)\end{array}$ & $\mathrm{p}<0.0001$ & $\chi^{2}(2)=5.81$ & $=0.055(0.127)$ & $x^{2}(1)=3.9$ & $=0.047(0.204)$ \\
\hline Don’t know & $7(1.1)$ & $9(0.5)$ & $0(0.0)$ & $1(0.5)$ & $0(0.0)$ & $0(0.0)$ \\
\hline Yes & 89 (13.5) & $122(6.5)$ & $28(22.4)$ & $28(12.8)$ & $21(26.6)$ & $18(15.1)$ \\
\hline No & $564(85.5)$ & $1748(93)$ & 97 (77.6) & $189(86.7)$ & $58(73.4)$ & $101(84.9)$ \\
\hline Diagnosed disease & $\begin{array}{l}\chi^{2}(1)=21.3 \\
(0.0001)\end{array}$ & $\mathrm{p}<0.0001$ & $\begin{array}{l}\chi^{2}(1)=8.01 \\
(0.023)\end{array}$ & $=0.005$ & $x^{2}(1)=2.1$ & $=0.143(0.485)$ \\
\hline Yes & $265(40.2)$ & $570(30.3)$ & $60(48)$ & $71(32.6)$ & $34(43)$ & $39(32.8)$ \\
\hline No & $395(59.9)$ & 1309 (69.7) & $65(52)$ & $147(67.4)$ & $45(57)$ & $80(67.2)$ \\
\hline
\end{tabular}

Table 5. Comorbidities in subjects with tinnitus with or without headache. Pairwise comparisons using Pearson's Chi-square test are reported. Percentages (\%) displayed refer to column percentages. P values adjusted for multiple comparisons are shown between parenthesis. Estimates in bold are statistically significant at 0.05 level.

Tinnitus phenotyping study. The online survey consisted of a combination of standardized questionnaires translated in Swedish ${ }^{40}$. Participants were asked the question: "Do you have tinnitus?" with possible answers being: "do not know", "no", "yes, occasionally (now and then)", "yes, always (all the time)". All of those answering "yes, occasionally" or "yes, always" were considered in this analysis and represent the "any tinnitus" group. The Tinnitus 
Sample Case History Questionnaire (TSCHQ) measures phenotypic characteristics that may be associated with tinnitus ${ }^{62}$, and question \#30 "Do you suffer from headache?" was adapted in Swedish leading to an intra-class coherent coefficient of 0.77 (good) in a test-retest ${ }^{40}$. The Tinnitus Handicap Inventory (THI) ${ }^{63}$, the Tinnitus Functional Index (TFI) ${ }^{64}$, the Fear of Tinnitus Questionnaire (FTQ ${ }^{65}$, the Tinnitus Catastrophizing Scale $(\mathrm{TCS})^{65}$, the Hyperacusis Questionnaire $(\mathrm{HQ})^{66}$, the Perceived Stress Questionnaire (PSQ) ${ }^{67}$, the Hospital Anxiety Depression Scales for Anxiety (HADS A) and depression (HADS D) ${ }^{68}$, and the World Health Organization's Quality of Life (WHOQoL)-BREF ${ }^{69}$ were used. Adaptation of these questionnaires to Swedish and their validity has been reported elsewehere ${ }^{40}$. Numerical Rating Scales (NRS) for Loudness, Awareness, and Annoyance were obtained via the TSCHQ (questions 12, 16, and 17). Severe tinnitus was operationally defined in two ways. The first used a revised grading system of the original eight-factor 25 item of the TFI ${ }^{70}$ where a TFI cut-off score $\geq 48$ denotes a big problem. The second used a THI cut-off score $\geq 58$, since this boundary is used as a criterion for referral to specialty care in the Stockholm County ${ }^{44}$, here referred to as severe tinnitus according to the terminology used in the THI. Importantly, these two groups both contain the most severe tinnitus cases according to two commonly used clinical tools. Overlap between the groups is expected. We present both groups to maximize comparability with other studies and minimize selection bias that could be introduced from choosing one tool for evaluation. For the present analysis we included 1,879 subjects with tinnitus without headache and 660 subjects with tinnitus with headache.

Statistical analysis. The statistical approach used in the tinnitus phenotyping study has been described elsewhere ${ }^{17}$. Briefly, phenotypic characteristics such as tinnitus loudness, pitch, onset, whether tinnitus is pulsating or not, what the tinnitus sounds like, and onset-related events were obtained from the TSCHQ. Additional sociodemographic data (i.e. marital status, income, employment status, and education level) were obtained using questions from Svensson et al. ${ }^{71}$. All statistical analyses were performed in JMP 13 (SAS Institute Inc.) and R (R Core Team, 2019). For nominal variables, Pearson's Chi-squared test was used. Homoscedasticity between groups was tested for using the Brown-Forsythe test and showed significant differences between subgroups for multiple variables. Multiple questionnaire total scores also deviated from a normal distribution. The non-parametric Wilcoxon's test and Cliffs delta $(\delta)$ for effect size, using the 'effsize' package in $\mathrm{R}$, were used for all comparisons to provide easy comparability between different groups for the reader. In order to investigate the potential impact of multiple comparisons on the discovery rates of our tests, we also report p-values adjusted by the method of Benjamini and Hochberg. The adjustments were computed for each set of $\mathrm{p}$-values resulting from multiple tests carried out.

For the association study, we estimated the odds ratios (ORs) and corresponding 95\% confidence intervals (CIs) for tinnitus using unconditional multiple logistic regression models after adjustment for sex, age, educational level (model 1) and also self-reported hearing ability (ESIT-SQ question A13) (model 2). Logistic regressions were performed in SAS 9.4 (SAS Institute, Cary, North Carolina).

\section{Data availability}

The datasets generated during and/or analysed during the current study are available from the corresponding author on reasonable request.

Received: 16 March 2020; Accepted: 4 May 2020;

Published online: 22 May 2020

\section{References}

1. McCormack, A., Edmondson-Jones, M., Somerset, S. \& Hall, D. A systematic review of the reporting of tinnitus prevalence and severity. Hearing Res. 337, 70-79, https://doi.org/10.1016/j.heares.2016.05.009 (2016).

2. Dobie, R. A. Depression and tinnitus. Otolaryngologic Clin. North. Am. 36, 383-388 (2003).

3. Bartels, H., Middel, B. L., van der Laan, B. F., Staal, M. J. \& Albers, F. W. The additive effect of co-occurring anxiety and depression on health status, quality of life and coping strategies in help-seeking tinnitus sufferers. Ear hearing 29, 947-956, https://doi. org/10.1097/AUD.0b013e3181888f83 (2008).

4. Kehrle, H. M., Sampaio, A. L., Granjeiro, R. C., de Oliveira, T. S. \& Oliveira, C. A. Tinnitus Annoyance in Normal-Hearing Individuals: Correlation With Depression and Anxiety. The. Ann. otology, rhinology, laryngology 125, 185-194, https://doi. org/10.1177/0003489415606445 (2016).

5. Hebert, S., Canlon, B. \& Hasson, D. Emotional exhaustion as a predictor of tinnitus. Psychotherapy Psychosom. 81, 324-326, https:// doi.org/10.1159/000335043 (2012).

6. Schlee, W. et al. Visualization of Global Disease Burden for the Optimization of Patient Management and Treatment. Front Med (Lausanne) 4:86, https://doi.org/10.3389/fmed.2017.00086 (2017).

7. Lugo, A. et al. Sex-specific influences of tinnitus on suicide attempt. JAMA Otolaryngol Head Neck Surg. 145(7), 685-687, https:// doi.org/10.1001/jamaoto.2019.0566 (2019).

8. Seo, J. H., Kang, J. M., Hwang, S. H., Han, K. D. \& Joo, Y. H. Relationship between tinnitus and suicidal behaviour in Korean men and women: a cross-sectional study. Clin. Otolaryngol. 41, 222-227, https://doi.org/10.1111/coa.12500 (2016).

9. Cederroth, C. R., Canlon, B. \& Langguth, B. Hearing loss and tinnitus-are funders and industry listening? Nat. Biotechnol. 31, 972-974, https://doi.org/10.1038/nbt.2736 (2013).

10. Cederroth, C. R., Dyhrfeld-Johnsen, J. \& Langguth, B. An update: emerging drugs for tinnitus. Expert Opin Emerg Drugs, 1-10, https://doi.org/10.1080/14728214.2018.1555240 (2018).

11. Langguth, B., Elgoyhen, A. B. \& Cederroth, C. R. Therapeutic Approaches to the Treatment of Tinnitus. Annu. Rev. Pharmacol. Toxicol. 59, 291-313, https://doi.org/10.1146/annurev-pharmtox-010818-021556 (2019).

12. Stockdale, D. et al. An economic evaluation of the healthcare cost of tinnitus management in the UK. BMC health Serv. Res. 17, 577, https://doi.org/10.1186/s12913-017-2527-2 (2017).

13. Cederroth, C. R. et al. Editorial: Towards an Understanding of Tinnitus Heterogeneity. Front. Aging Neurosci. 11, 53, https://doi. org/10.3389/fnagi.2019.00053 (2019).

14. Schlee, W. et al. Innovations in Doctoral Training and Research on Tinnitus: The European School on Interdisciplinary Tinnitus Research (ESIT) Perspective. Front. Aging Neurosci. 9, 447, https://doi.org/10.3389/fnagi.2017.00447 (2017). 
15. Schecklmann, M., Landgrebe, M., Langguth, B. \& Group, T. R. I. D. S. Phenotypic characteristics of hyperacusis in tinnitus. PLoS One 9, e86944, https://doi.org/10.1371/journal.pone.0086944 (2014).

16. Vielsmeier, V. et al. Temporomandibular joint disorder complaints in tinnitus: further hints for a putative tinnitus subtype. Plos One 7, e38887, https://doi.org/10.1371/journal.pone.0038887 (2012).

17. Edvall, N. K. et al. Impact of Temporomandibular Joint Complaints on Tinnitus-Related Distress. Front. Neurosci. 13, 879, https:// doi.org/10.3389/fnins.2019.00879 (2019).

18. Langguth, B., Hund, V., Landgrebe, M. \& Schecklmann, M. Tinnitus Patients with Comorbid Headaches: The Influence of Headache Type and Laterality on Tinnitus Characteristics. Front. Neurol. 8, 440, https://doi.org/10.3389/fneur.2017.00440 (2017).

19. Burstein, R., Noseda, R. \& Borsook, D. Migraine: multiple processes, complex pathophysiology. J. neuroscience: Off. J. Soc. Neurosci. 35, 6619-6629, https://doi.org/10.1523/JNEUROSCI.0373-15.2015 (2015).

20. Brunnberg, E., Linden-Bostrom, M. \& Berglund, M. Tinnitus and hearing loss in 15-16-year-old students: mental health symptoms, substance use, and exposure in school. Int. J. audiology 47, 688-694, https://doi.org/10.1080/14992020802233915 (2008).

21. Kim, Y. H. et al. Tinnitus in children: association with stress and trait anxiety. Laryngoscope 122, 2279-2284, https://doi.org/10.1002/ lary.23482 (2012)

22. Jaramillo, C. A. et al. A cohort study examining headaches among veterans of Iraq and Afghanistan wars: Associations with traumatic brain injury, PTSD, and depression. Headache 56, 528-539, https://doi.org/10.1111/head.12726 (2016).

23. Pezzoli, M. et al. Tinnitus and its relationship with muscle tenderness in patients with headache and facial pain. J. laryngology otology 129, 638-643, https://doi.org/10.1017/S0022215115001425 (2015).

24. Guichard, E., Montagni, I., Tzourio, C. \& Kurth, T. Association Between Headaches and Tinnitus in Young Adults: Cross-Sectional Study. Headache 56, 987-994, https://doi.org/10.1111/head.12845 (2016).

25. Sindhusake, D. et al. Risk factors for tinnitus in a population of older adults: the blue mountains hearing study. Ear hearing 24, 501-507, https://doi.org/10.1097/01.AUD.0000100204.08771.3D (2003).

26. Hwang, J. H., Tsai, S. J., Liu, T. C., Chen, Y. C. \& Lai, J. T. Association of Tinnitus and Other Cochlear Disorders With a History of Migraines. JAMA Otolaryngol. Head. Neck Surg. 144, 712-717, https://doi.org/10.1001/jamaoto.2018.0939 (2018).

27. Shore, S. E., Roberts, L. E. \& Langguth, B. Maladaptive plasticity in tinnitus-triggers, mechanisms and treatment. Nat. reviews. Neurol. 12, 150-160, https://doi.org/10.1038/nrneurol.2016.12 (2016).

28. Auerbach, B. D., Rodrigues, P. V. \& Salvi, R. J. Central gain control in tinnitus and hyperacusis. Front. Neurol. 5, 206, https://doi. org/10.3389/fneur.2014.00206 (2014).

29. Rauschecker, J. P., Leaver, A. M. \& Muhlau, M. Tuning out the noise: limbic-auditory interactions in tinnitus. Neuron 66, 819-826, https://doi.org/10.1016/j.neuron.2010.04.032 (2010).

30. Rauschecker, J. P., May, E. S., Maudoux, A. \& Ploner, M. Frontostriatal Gating of Tinnitus and Chronic Pain. Trends Cognit. Sci. 19, 567-578, https://doi.org/10.1016/j.tics.2015.08.002 (2015)

31. Schlee, W., Weisz, N., Bertrand, O., Hartmann, T. \& Elbert, T. Using auditory steady state responses to outline the functional connectivity in the tinnitus brain. Plos One 3, e3720, https://doi.org/10.1371/journal.pone.0003720 (2008).

32. De Ridder, D., Vanneste, S., Langguth, B. \& Llinas, R. Thalamocortical Dysrhythmia: A Theoretical Update in Tinnitus. Front. Neurol. 6, 124, https://doi.org/10.3389/fneur.2015.00124 (2015).

33. de Tommaso, M. et al. Altered processing of sensory stimuli in patients with migraine. Nature reviews. Neurology 10, 144-155, https://doi.org/10.1038/nrneurol.2014.14 (2014).

34. Vanneste, S., Song, J. J. \& De Ridder, D. Thalamocortical dysrhythmia detected by machine learning. Nat. Commun. 9, 1103, https:// doi.org/10.1038/s41467-018-02820-0 (2018).

35. Akerman, S. \& Romero-Reyes, M. Insights into the pharmacological targeting of the trigeminocervical complex in the context of treatments of migraine. Expert. Rev. Neurother. 13, 1041-1059, https://doi.org/10.1586/14737175.2013.827472 (2013).

36. May, A. Diagnosis and clinical features of trigemino-autonomic headaches. Headache 53, 1470-1478, https://doi.org/10.1111/ head.12213 (2013).

37. Haider, H. F. et al. Pathophysiology, Diagnosis and Treatment of Somatosensory Tinnitus: A Scoping Review. Front. Neurosci. 11, 207, https://doi.org/10.3389/fnins.2017.00207 (2017).

38. Kim, S. Y., Jeon, Y. J., Lee, J.-Y. \& Kim, Y. H. In The Laryngoscope Vol. 127 2113-2119 (Wiley-Blackwell, 2017).

39. Swan, A. A. et al. In Hearing research Vol. 349 04-dic (2017).

40. Müller, K. et al. Validation of Online Versions of Tinnitus Questionnaires Translated into Swedish. Front. Aging Neurosci. 8, 272, https://doi.org/10.3389/fnagi.2016.00272 (2016).

41. IHS. Headache Classification Committee of the International Headache Society (IHS) The International Classification of Headache Disorders, 3rd edition. Cephalalgia: an international journal of headache 38, 1-211, https://doi.org/10.1177/0333102417738202 (2018).

42. Lempert, T. et al. Vestibular migraine: diagnostic criteria. J. Vestib. Res. 22, 167-172, https://doi.org/10.3233/VES-2012-0453 (2012).

43. Sindhusake, D. et al. Prevalence and characteristics of tinnitus in older adults: the Blue Mountains Hearing Study. Int. J. audiology 42, 289-294 (2003)

44. Idrizbegovic, E., Kjerulf, E. \& Team for Diagnostics Hearing Habilitation Children and Youth and Hearing Rehabilitation for Adults. Tinnitus Care Program [Tinnitus Vårdprogram] (ed. Karolinska Sjukhuset) (Stockholm, 2011).

45. Lopez-Escamez, J. A. et al. Accompanying Symptoms Overlap during Attacks in Meniere's Disease and Vestibular Migraine. Front. Neurol. 5, 265, https://doi.org/10.3389/fneur.2014.00265 (2014).

46. Eggers, S. D., Neff, B. A., Shepard, N. T. \& Staab, J. P. Comorbidities in vestibular migraine. J. Vestib. Res. 24, 387-395, https://doi. org/10.3233/VES-140525 (2014).

47. Langguth, B. et al. Tinnitus and Headache. BioMed. Res. Int. 2015, 797416, https://doi.org/10.1155/2015/797416 (2015).

48. Maas, I. L. et al. Genetic susceptibility to bilateral tinnitus in a Swedish twin cohort. Genet. medicine: Off. J. Am. Coll. Med. Genet. 19, 1007-1012, https://doi.org/10.1038/gim.2017.4 (2017).

49. Cederroth, C. R. et al. Association of Genetic vs Environmental Factors in Swedish Adoptees With Clinically Significant Tinnitus. JAMA Otolaryngol Head Neck Surg, https://doi.org/10.1001/jamaoto.2018.3852 (2019).

50. Yu, H. et al. GLAST Deficiency in Mice Exacerbates Gap Detection Deficits in a Model of Salicylate-Induced Tinnitus. Frontiers in Behavioral Neuroscience 10, https://doi.org/10.3389/fnbeh.2016.00158 (2016)

51. Gormley, P. et al. Meta-analysis of 375,000 individuals identifies 38 susceptibility loci for migraine. Nat. Genet. 48, 856-866, https:// doi.org/10.1038/ng.3598 (2016).

52. Vona, B., Nanda, I., Shehata-Dieler, W. \& Haaf, T. Genetics of Tinnitus: Still in its Infancy. Front. Neurosci. 11, 236, https://doi. org/10.3389/fnins.2017.00236 (2017).

53. Lopez-Escamez, J. A. et al. Genetics of tinnitus: an emerging area for molecular diagnosis and drug development. Frontiers in Neuroscience 10, https://doi.org/10.3389/fnins.2016.00377 (2016)

54. Cederroth, C. R., Kähler, A., Sullivan, P. F. \& Lopez-Escamez, J. A. Genetics of tinnitus: time to biobank phantom sounds. Front. Genet. 8, 110, https://doi.org/10.3389/fgene.2017.00110 (2017).

55. Szczepek, A. J. et al. Recommendations on Collecting and Storing Samples for Genetic Studies in Hearing and Tinnitus Research. Ear hearing 40, 219-226, https://doi.org/10.1097/AUD.0000000000000614 (2019). 
56. Bueno, C. H., Pereira, D. D., Pattussi, M. P., Grossi, P. K. \& Grossi, M. L. Gender differences in temporomandibular disorders in adult populational studies: A systematic review and meta-analysis. J. Oral. Rehabil. 45, 720-729, https://doi.org/10.1111/joor.12661 (2018).

57. Leonardi, M., Steiner, T. J., Scher, A. T. \& Lipton, R. B. The global burden of migraine: measuring disability in headache disorders with WHO's Classification of Functioning. Disability Health . J. Headache Pain. 6, 429-440, https://doi.org/10.1007/s10194-0050252-4 (2005).

58. Nordic Burden of Disease, C. Life expectancy and disease burden in the Nordic countries: results from the Global Burden of Diseases, Injuries, and Risk Factors Study 2017. Lancet Public Health 4, e658-e669, https://doi.org/10.1016/S2468-2667(19)30224-5 (2019).

59. Seydel, C., Haupt, H., Olze, H., Szczepek, A. J. \& Mazurek, B. Gender and chronic tinnitus: differences in tinnitus-related distress depend on age and duration of tinnitus. Ear hearing 34, 661-672, https://doi.org/10.1097/AUD.0b013e31828149f2 (2013).

60. Almqvist, C. et al. LifeGene-a large prospective population-based study of global relevance. Eur. J. Epidemiol. 26, 67-77, https://doi. org/10.1007/s10654-010-9521-x (2011).

61. Genitsaridi, E. et al. Standardised profiling for tinnitus research: The European School for Interdisciplinary Tinnitus Research Screening Questionnaire (ESIT-SQ). Hearing Res. https://doi.org/10.1016/j.heares.2019.02.017 (2019).

62. Landgrebe, M. et al. The Tinnitus Research Initiative (TRI) database: a new approach for delineation of tinnitus subtypes and generation of predictors for treatment outcome. BMC Med. Inform. Decis. Mak. 10, 42, https://doi.org/10.1186/1472-6947-10-42 (2010).

63. Newman, C. W., Jacobson, G. P. \& Spitzer, J. B. Development of the Tinnitus Handicap Inventory. Arch. Otolaryngol. Head. Neck Surg. 122, 143-148 (1996).

64. Meikle, M. B. et al. The tinnitus functional index: development of a new clinical measure for chronic, intrusive tinnitus. Ear hearing 33, 153-176, https://doi.org/10.1097/AUD.0b013e31822f67c0 (2012).

65. Cima, R. F., Crombez, G. \& Vlaeyen, J. W. Catastrophizing and fear of tinnitus predict quality of life in patients with chronic tinnitus. Ear hearing 32, 634-641, https://doi.org/10.1097/AUD.0b013e31821106dd (2011).

66. Khalfa, S. et al. Psychometric normalization of a hyperacusis questionnaire. ORL; journal for oto-rhino-laryngology and its related specialties 64, 436-442, doi:67570 (2002).

67. Levenstein, S. et al. Development of the Perceived Stress Questionnaire: a new tool for psychosomatic research. J. Psychosom. Res. 37, 19-32 (1993).

68. Andersson, G., Kaldo-Sandstrom, V., Strom, L. \& Stromgren, T. Internet administration of the Hospital Anxiety and Depression Scale in a sample of tinnitus patients. J. Psychosom. Res. 55, 259-262 (2003).

69. The WHOQOL Group. Development of the World Health Organization WHOQOL-BREF quality of life assessment.. Psychological medicine 28, 551-558 (1998).

70. Fackrell, K., Hall, D. A., Barry, J. G. \& Hoare, D. J. Performance of the Tinnitus Functional Index as a diagnostic instrument in a UK clinical population. Hearing Res. https://doi.org/10.1016/j.heares.2017.10.016 (2017).

71. Svensson, A. C. et al. Cohort profile: The Stockholm Public Health Cohort. Int. J. Epidemiol. 42, 1263-1272, https://doi.org/10.1093/ ije/dys126 (2013).

\section{Acknowledgements}

We gratefully acknowledge the support and generosity of Nancy Pedersen, head of LifeGene. This study was funded by the GENDER-NET Co-Plus Fund (GNP-182, to CRC, SG, JALE and JB). In addition, CRC has received research funding from Decibel Therapeutics, Inc., Svenska Läkaresällskapet (SLS-779681), Tysta Skolan, Hörselforskningsfonden (\#503), the European Union's Horizon 2020 research and innovation programme under the Marie Skłodowska-Curie grant agreement No. 72204655. BC has received funding from the Swedish Medical Research Council K2014-99×-22478-01-3, Karolinska Institutet and Tysta Skolan. SG is an Honorary Associate Professor at the University of Nottingham, School of Medicine. Open access funding provided by Karolinska Institute.

\section{Author contributions}

C.R.C., S.G. and B.C. designed the study. N.K.E. collected, extracted, and processed the STOP data. N.K.E. and A.Lu. analyzed the data and prepared all tables. A.La., G.M., J.A.L.E., J.B. and I.U. helped to develop the scientific arguments and contributed to data interpretation. All authors played a role in writing the manuscript and approved the final version.

\section{Competing interests}

G.M. is an employee of Decibel Therapeutics. All other authors declare no competing interests.

\section{Additional information}

Supplementary information is available for this paper at https://doi.org/10.1038/s41598-020-65395-1.

Correspondence and requests for materials should be addressed to C.R.C.

Reprints and permissions information is available at www.nature.com/reprints.

Publisher's note Springer Nature remains neutral with regard to jurisdictional claims in published maps and institutional affiliations.

(c) (i) Open Access This article is licensed under a Creative Commons Attribution 4.0 International (c) License, which permits use, sharing, adaptation, distribution and reproduction in any medium or format, as long as you give appropriate credit to the original author(s) and the source, provide a link to the Creative Commons license, and indicate if changes were made. The images or other third party material in this article are included in the article's Creative Commons license, unless indicated otherwise in a credit line to the material. If material is not included in the article's Creative Commons license and your intended use is not permitted by statutory regulation or exceeds the permitted use, you will need to obtain permission directly from the copyright holder. To view a copy of this license, visit http://creativecommons.org/licenses/by/4.0/.

(C) The Author(s) 2020 